\title{
Novel Data Assimilation Algorithm for Nearshore Bathymetry
}

\author{
HOJAT GHORBANIDEHNO \\ Mechanical Engineering Department, Stanford University, Stanford, California \\ JONGHYUN LEE \\ Department of Civil and Environmental Engineering, and Water Resources Research Center, \\ University of Hawai'i at Mānoa, Honolulu, Hawaii \\ MATTHEW FARTHING AND TYLER HESSER \\ Coastal and Hydraulics Laboratory, U.S. Army Engineer Research and Development Center, \\ Vicksburg, Mississippi \\ PETER K. KITANIDIS \\ Department of Civil and Environmental Engineering, Stanford University, \\ Stanford, California \\ ERIC F. DARVE \\ Mechanical Engineering Department, Stanford University, Stanford, California
}

(Manuscript received 27 April 2018, in final form 29 January 2019)

\begin{abstract}
It can be expensive and difficult to collect direct bathymetry data for nearshore regions, especially in highenergy locations where there are temporally and spatially varying bathymetric features like sandbars. As a result, there has been increasing interest in remote assessment techniques for estimating bathymetry. Recent efforts have combined Kalman filter-based techniques with indirect video-based observations for bathymetry inversion. Here, we estimate nearshore bathymetry by utilizing observed wave celerity and wave height, which are related to bathymetry through phase-averaged wave dynamics. We present a modified compressed-state Kalman filter (CSKF) method, a fast and scalable Kalman filter method for linear and nonlinear problems with large numbers of unknowns and measurements, and apply it to two nearshore bathymetry estimation problems. To illustrate the robustness and accuracy of our method, we compare its performance with that of two ensemble-based approaches on twin bathymetry estimation problems with profiles based on surveys taken by the U.S. Army Corps of Engineer Field Research Facility (FRF) in Duck, North Carolina. We first consider an estimation problem for a temporally constant bathymetry profile. Then we estimate bathymetry as it evolves in time. Our results indicate that the CSKF method is more accurate and robust than the ensemble-based methods with the same computational cost. The superior performance is due to the optimal low-rank representation of the covariance matrices.
\end{abstract}

\section{Introduction}

Nearshore bathymetry may change on a wide range of spatial and temporal scales due to the often strong interaction of waves, currents, and a mobile bottom. Shipboard sounding and other standard in situ bathymetry measurements are slow, expensive, and occasionally even

Corresponding author: Hojat Ghorbanidehno, hotjjatgh@stanford.edu hazardous. In situ measurement techniques are extremely difficult during periods of large waves when significant bathymetry changes are likely to occur. Furthermore, technological restrictions limit the spatial and temporal coverage attainable with in situ measurements in the nearshore (Tronvig 2005). Therefore, remote sensing techniques are valuable tools to monitor bathymetry changes in the nearshore zone (Holman and Haller 2013; Stockdon and Holman 2000). 
Several remote sensing methods have been suggested to estimate nearshore bathymetry. For clear water where the bottom is visible, methods based on light penetration in the water column and its reflection of the seabed can be used to estimate bathymetry (Mobley et al. 2005; Irish and Lillycrop 1999; Sallenger et al. 2003; Lyzenga et al. 2006) including multispectral or hyperspectral (Mobley et al. 2005; Benny and Dawson 1983; Bierwirth et al. 1993; Sandidge and Holyer 1998) and light detection and ranging technology (Irish and Lillycrop 1999; Lyzenga 1985; Liu et al. 2007). However, for many areas of interest, the bottom is often not visible due to, among other things, turbidity and breaking waves. In these situations, depth must be estimated through the use of ocean surface observables (Aarninkhof et al. 2005). Image processing techniques have been used to get quantitative hydrodynamic observations from ocean surface images (Holland et al. 2001; De Vries et al. 2011; Puleo et al. 2003). Although these techniques can provide broad spatial and temporal measurements they have high measurement uncertainty (noise) (Holman et al. 2013; Brodie et al. 2018). Data assimilation techniques can be used to extract the information contained in the noisy observations and combine them with a physics-based model to achieve an accurate estimate of bathymetry and a quantification of its uncertainty.

Several data assimilation methods have been used to incorporate surface observations such as currents, wave celerity, and wave heights in inversion modeling for bathymetry estimation (Holman and Haller 2013; Van Dongeren et al. 2008; Sasso 2012; Holman et al. 1991; Morris 2013). For example, Holman et al. (2013) has assimilated pseudo-observations of depth (i.e., observations derived from an explicit depth inversion) from remote sensing by using a Kalman filter (KF) method for each grid point (Kalman 1960; Pham et al. 1998). In this method, the spatial covariance of the state noise and the prior state are neglected but despite this simplification, results may be obtained that are consistent with directly measured bathymetry data. This consistency stems from having spatially dense observations and acknowledging data error as well as model error. However, neglecting the spatial covariance means that only a local correction is provided; instead, one single observation, if relevant, should also correct neighboring grid points (Wilson et al. 2014, 2010). To tackle this problem, the full covariance matrix should be considered in the Kalman filter method. However, including the full covariance matrix in this method increases its computational cost for high-dimensional problems significantly (Ghorbanidehno et al. 2015; Kitanidis 2015). This "curse of dimensionality" becomes further pronounced when the bathymetry problem in question extends over large-scale domains.
For such problems with a large state and parameter spaces, applying the textbook Kalman filter or extended Kalman filter (EKF) is practically impossible because it requires updating large covariance matrices and constructing Jacobian matrices at each data assimilation step. Specifically, constructing the Jacobian matrices for large-scale problems requires a large number of simulation model runs proportional to the number of observations or the number of unknowns, which would be the most expensive part in the bathymetry problems considered here. Therefore, several techniques such as low-rank approximation ( $\mathrm{Li}$ et al. 2015; Kitanidis 2015), upscaling (Tahmasebi and Sahimi 2018; Möhrlen and Jørgensen 2009; Tahmasebi 2017; Tahmasebi and Kamrava 2018), or ensemblebased techniques (Evensen 2003, 2009) have been used to reduce these computational cost issues.

Ensemble-based Kalman filters (Evensen 2003; Houtekamer and Mitchell 2005; Evensen 2009; Ott et al. 2004) are among the most widely used fast Kalman filter variants for large-scale problems. Recently, these methods have been used for large-scale bathymetry problems (Van Dongeren et al. 2008; Wilson and Özkan-Haller 2012; Wilson et al. 2014, 2010). Ensemble-based methods are reduced-order methods and thus are computationally more efficient than the Kalman filter and the extended Kalman filter for spatially dense covariance matrices; however, for strongly nonlinear problems these methods may compute model parameters or state variables that are unphysical or inaccurate (Tahmasebi et al. 2018; Lee et al. 2018; Tahmasebi and Sahimi 2013; Li et al. 2015), and they typically suffer from spurious model correlation and ensemble spread reduction (Anderson 2001; Hamill and Snyder 2000; Hunt et al. 2007). Also, it has been shown that ensemble-based methods may require many realizations for problems with a large number of observations (Ghorbanidehno et al. 2015, 2017; Li et al. 2015; Kepert 2004).

In this paper, we present the modified compressedstate Kalman filter (CSKF), a fast and scalable extended Kalman filter, and demonstrate the applicability of this method to large-scale bathymetry estimation problems with a large number of measurements. The core idea behind the computational efficiency of this method is to provide an accurate low-rank representation of the covariance matrix (Kitanidis 2015) and to avoid explicit calculation of the Jacobian matrices by using an efficient matrix-free approach (Ghorbanidehno et al. 2015; Kitanidis and Lee 2014; Lee and Kitanidis 2014). Here we extend the original CSKF algorithm and incorporate the Sherman-Morrison-Woodbury formula to improve performance for large numbers of observations. 
CSKF estimates spatially distributed unknown parameters and quantifies the corresponding uncertainty rigorously in a Bayesian framework (Kitanidis 2015) and has been applied successfully to high-dimensional problems with a small number of data (Kitanidis 2015; Li et al. 2015, 2017) but this is the first time that CSKF is applied to a high-dimensional, data-intensive problem.

CSKF and ensemble-based methods both employ lowrank approximations of the covariance matrices to reduce the computational cost. However, CSKF uses the eigendecomposition of covariance matrices, which is the optimal low-rank approximation (Kitanidis 2015) while ensemble-based methods sample from the covariance matrices to construct a low-rank representation. Therefore, the actual rank of ensemble-based methods may be smaller than the ensemble size due to intrasample multicollinearity introduced by the pseudorandom sampler and numerical error (Lee et al. 2018). This issue can lead to the development of long-range spurious correlations between observations and state or parameter variables as shown in Li et al. (2015). Additionally, Li et al. (2015) demonstrated that CSKF can provide more accurate results than ensemble-based methods for strongly nonlinear problems by applying these techniques to a $\mathrm{CO}_{2}$ monitoring problem where there is nonlinearity in both the model and the observation process.

The paper is organized as follows. We start with a brief presentation of the extended Kalman filter to establish the notation, followed by the algorithm and the implementation details of the modified CSKF method in section 2 . Then, in sections 3 and 4 , we evaluate the accuracy and robustness of this method in two bathymetry test cases. In the first, we estimate bathymetry for a problem with temporally constant bathymetry profile using noisy wave celerity and wave height measurements. Then, in the second one, we consider a more challenging nonsteady problem with small-scale bathymetry features and large-scale temporal evolution between remote measurements where we only have access to noisy wave celerity measurements. For both problems, we compare the results of CSKF with those obtained by ensemble-based approaches (Wilson et al. 2014). By doing so, we will show that our method performs very well for high-dimensional, data-intensive problems in an efficient way without loss of accuracy, and thus is suitable for future nearshore bathymetry estimation problems.

\section{Methods}

This section presents the formulation of the bathymetry estimation problem as a dynamic data assimilation problem solved through Kalman filtering techniques.
We summarize the solution by the extended Kalman filter (Nenna et al. 2011; Anderson and Moore 1979) to establish the notation and then we present the modified CSKF algorithm.

\section{a. Extended Kalman filter}

Consider a nonlinear dynamical system with $m$ number of unknowns in a state vector denoted by $\mathbf{x}_{t}$ in $\mathbf{R}^{m}$ as

$$
\mathbf{x}_{t+1}=f\left(\mathbf{x}_{t}\right)+\mathbf{w}_{t},
$$

where $f(\cdots)$ is the forward transition model which propagates the state vector $\mathbf{x}_{t}$ from time $t$ to time $t+1$; $\mathbf{w}_{t}$ is the model error or noise and is modeled as a multivariate Gaussian random vector with zero mean and with covariance matrix $\mathbf{Q}_{t}$. The measurements are related to the state variable as

$$
\mathbf{y}_{t}=h\left(\mathbf{x}_{t+1}\right)+\mathbf{v}_{t}, \quad \mathbf{y} \in \mathbf{R}^{n},
$$

where $\mathbf{y}_{t}$ is the observation at time step $t, h(\cdots)$ is the observation model, $n$ is the number of measurements, and $\mathbf{v}_{t} \sim N\left(0, \mathbf{R}_{t}\right)$ represent the noise in the measurement vector.

For the above state-space models, the EKF gives the posterior mean $\hat{\mathbf{x}}_{t+1 \mid t+1}$ and estimation error covariance matrix $\mathbf{P}_{t+1 \mid t+1}$ conditioned on the observations $\mathbf{y}_{\mathbf{t}+1}$ in the following algorithm:

$$
\begin{aligned}
\hat{\mathbf{x}}_{t+1 \mid t} & =f\left(\mathbf{x}_{t \mid t}\right), \\
\mathbf{P}_{t+1 \mid t} & =\mathbf{F}_{t} \mathbf{P}_{t \mid t} \mathbf{F}_{t}^{\mathrm{T}}+\mathbf{Q}_{t}, \\
\mathbf{K}_{t+1} & =\mathbf{P}_{t+1 \mid t} \mathbf{H}_{t}^{\mathrm{T}}\left(\mathbf{H}_{t} \mathbf{P}_{t+1 \mid t} \mathbf{H}_{t}^{\mathrm{T}}+\mathbf{R}_{t}\right)^{-1}, \\
\hat{\mathbf{x}}_{t+1 \mid t+1} & =\hat{\mathbf{x}}_{t+1 \mid t}+\mathbf{K}_{t+1}\left[\mathbf{y}_{t+1}-h\left(\hat{\mathbf{x}}_{t+1 \mid t}\right)\right], \\
\mathbf{P}_{t+1 \mid t+1} & =\mathbf{P}_{t+1 \mid t}-\mathbf{K}_{t+1} \mathbf{H}_{t} \mathbf{P}_{t+1 \mid t},
\end{aligned}
$$

where $\mathbf{K}$ is the Kalman gain matrix, $\mathbf{P}_{t+1}$ is the estimation error covariance matrix, and $\mathbf{F}$ and $\mathbf{H}$ in all equations are the Jacobian matrices of the forward model and measurement model, respectively (Ghorbanidehno et al. 2015; Khodayi-mehr et al. 2018b):

$$
\mathbf{F}_{t}=\left.\frac{\partial f}{\partial \mathbf{x}}\right|_{t} \quad \text { and }\left.\quad \mathbf{H}_{t} \frac{\partial h}{\partial \mathbf{x}}\right|_{t} .
$$

Although the EKF provides accurate results in the least squares errors sense, the implementation of this algorithm for high-dimensional, data-intense problems becomes infeasible because of the high storage cost and computational cost for matrix-matrix products and for obtaining the Jacobian matrices $\mathbf{F}$ and $\mathbf{H}$. The next 
TABLE 1. Comparison of the computational cost of the CSKF and the EnKF for nonlinear problems measured in terms of the number of forward model calls, $f(\cdots)$ and $h(\cdots)$, and the cost of matrix operations; $N$ is the ensemble size, $n$ is number of measurements, $m$ is number of unknowns, and $r$ is effective rank of the $\mathbf{Q}$ matrix.

\begin{tabular}{|c|c|}
\hline Operation & $\mathscr{Q}($ Operation) \\
\hline \multicolumn{2}{|l|}{ CSKF } \\
\hline $\mathbf{x}_{t+1}^{f}=f\left(\mathbf{x}_{t}^{a}\right)$ & $1 f(\cdots)$ \\
\hline $\mathbf{C}_{t+1}^{f}=\mathbf{A}^{\mathrm{T}}\left(\mathbf{F}_{t} \mathbf{A}\right) \mathbf{C}_{t}^{a}\left(\mathbf{F}_{t} \mathbf{A}\right)^{\mathrm{T}} \mathbf{A}+\mathbf{D}_{t}$ & $r f(\cdots)+r^{2} m$ \\
\hline $\mathbf{K}_{t+1}=\mathbf{A} \mathbf{C}_{t+1}^{f}\left(\mathbf{H}_{t} \mathbf{A}\right)^{\mathrm{T}}\left\{\mathbf{R}_{t}^{-1}-\mathbf{R}_{t}^{-1}\left(\mathbf{H}_{t} \mathbf{A}\right)\left[\mathbf{C}_{t+1}^{-f}\left(\mathbf{H}_{t} \mathbf{A}\right)^{\mathrm{T}} \mathbf{R}_{t}^{-1}\left(\mathbf{H}_{t} \mathbf{A}\right)\right]^{-1}\left(\mathbf{H}_{t} \mathbf{A}\right)^{\mathrm{T}} \mathbf{R}_{t}^{-1}\right\}$ & $r h(\cdots)+r^{2} m+r^{3}+r n m$ \\
\hline $\mathbf{x}_{t+1}^{a}=\mathbf{x}_{t+1}^{f}+\mathbf{K}_{t+1}\left[\mathbf{y}_{t+1}-h\left(\mathbf{x}_{t+1}^{f}\right)\right]$ & $n m+r^{2} n+1 f(\cdots)$ \\
\hline $\mathbf{C}_{t+1}^{a}=\mathbf{C}_{t+1}^{f}-\mathbf{A}^{\mathrm{T}} \mathbf{K}_{t+1}\left(\mathbf{H}_{t} \mathbf{A}\right) \mathbf{C}_{t+1}^{f}$ & $r^{2}+n m^{2}$ \\
\hline \multicolumn{2}{|l|}{ EnKF } \\
\hline $\mathbf{x}_{t+1, j}^{f}=f_{t+1}\left(\mathbf{x}_{t, j}^{a}\right)+\mathbf{w}_{t+1, j}, \quad j=1, \ldots, N$ & $N f(\cdots)$ \\
\hline $\mathbf{D}_{t+1}=\left[\mathbf{x}_{t+1,1}^{f}-\overline{\mathbf{x}}_{t+1}^{f}, \ldots, \mathbf{x}_{t+1, N}^{f}-\overline{\mathbf{x}}_{t+1}^{f}\right]$ & $N m$ \\
\hline $\mathbf{P}_{t+1}^{f}=\frac{1}{N-1} \mathbf{D}_{t+1} \mathbf{D}_{t+1}^{\mathrm{T}}$ & $N^{2} m$ \\
\hline $\mathbf{K}_{t+1}=\mathbf{P}_{t+1}^{f} \mathbf{H}^{\mathrm{T}}\left(\mathbf{H P}_{t+1}^{f} \mathbf{H}^{\mathrm{T}}+\mathbf{R}\right)^{-1}$ & $N h(\cdots)+N n m+n^{3}+N n^{2}$ \\
\hline $\mathbf{x}_{t+1, j}^{a}=\mathbf{s}_{t+1, j}^{f}+\mathbf{K}_{t+1}\left(\mathbf{y}_{t+1}-\mathbf{H} \mathbf{x}_{t+1, j}^{f}+\mathbf{v}_{t+1, j}\right)$ & Nnm \\
\hline $\mathbf{x}_{t+1}^{a}=\frac{1}{N} \sum_{j=1}^{N} \mathbf{x}_{t+1, j}^{a}$ & $\mathrm{Nm}$ \\
\hline
\end{tabular}

section outlines how these operations can be made more efficiently.

\section{b. Compressed-state Kalman filter}

Low-rank approximation can be used to reduce the cost of storing and updating the covariance matrices. $\mathrm{Li}$ et al. (2015), Kitanidis (2015), Khodayi-mehr et al. (2017), and Pham et al. (1998) used covariance compression techniques to approximate the covariance matrix $\mathbf{P}_{t}$ with a lowrank matrix. This process can be shown as below:

$$
\mathbf{P}_{t} \approx \mathbf{A C}_{t} \mathbf{A}^{\mathrm{T}},
$$

where $\mathbf{A} \in \mathbf{R}^{m \times r}$ contains the orthogonal basis and satisfies $\mathbf{A} \mathbf{A}^{\mathrm{T}}=\mathbf{I}$ and $\mathbf{C}_{t} \in \mathbf{R}^{r \times r}$ is a small symmetric positive definite matrix. The columns of $\mathbf{A}$ are orthonormal vectors that span a subspace of the full $m$-dimensional space matrix selected for high compressive power; that is, the projection of the actual state on this subspace is a good approximation of the actual state. There are several methods to choose the $r$ columns of $\mathbf{A}$. For example, A can be computed using direct eigendecomposition with computational complexity of $\mathscr{O}\left(\mathrm{m}^{3}\right)$. However, since only the first $r$ dominant eigenvectors are required, they can be computed at much lower cost using computationally more efficient techniques such as randomized low-rank approximation (Halko et al. 2011). Especially, for problems discretized on a regular grid, the discrete cosine transform (DCT) (Ahmed et al. 1974; Kitanidis 2015; Strang 1999; Rao and Yip 2014) is an established and computationally advantageous method for generating an orthonormal basis $A$ (Strang 1999; Kitanidis 2015; Li et al. 2015) with the computational complexity $\mathscr{O}(m r)$. We used the DCT method in this paper to compute the orthonormal basis and as suggested by (Kitanidis 2015), we used the same basis for both $\mathbf{P}$ and $\mathbf{Q}$ matrices and we kept the preselected basis constant for all data assimilation steps. Another important part of our method is the calculation of the Jacobian matrices $\mathbf{F}$ and $\mathbf{H}$ and then computing the matrix-matrix products of them with matrix $\mathbf{A}$. As previously mentioned, both of these steps are computationally expensive for large-scale problems. To overcome this problem, we used a matrix-free approach (Ghorbanidehno et al. 2015; Kitanidis and Lee 2014). This method directly computes the product of the Jacobian with a vector $\mathbf{x}$ instead of calculating the Jacobian matrix and then multiplying it with $\mathbf{x}$. Consider we want to compute $\mathbf{F} \mathbf{x}$ and $\mathbf{H x}$, where $\mathbf{F}=\partial f / \partial \mathbf{s}, \mathbf{H}=\partial h / \partial \mathbf{s}$ at $\mathbf{s}=\mathbf{s}_{0}$, and $\mathbf{x}$ is a vector. Then $\mathbf{F x}$ and $\mathbf{H x}$ are computed directly by

$$
\begin{aligned}
& \mathbf{F} \mathbf{x} \simeq \frac{\|\mathbf{x}\|}{\delta\left\|\mathbf{s}_{0}\right\|}\left[f\left(\mathbf{s}_{0}+\frac{\left\|\mathbf{s}_{0}\right\|}{\|\mathbf{x}\|} \delta \mathbf{x}\right)-f\left(\mathbf{s}_{0}\right)\right], \\
& \mathbf{H} \mathbf{x} \simeq \frac{\|\mathbf{x}\|}{\delta\left\|\mathbf{s}_{0}\right\|}\left[h\left(\mathbf{s}_{0}+\frac{\left\|\mathbf{s}_{0}\right\|}{\|\mathbf{x}\|} \delta \mathbf{x}\right)-h\left(\mathbf{s}_{0}\right)\right],
\end{aligned}
$$

where the ratio of Euclidean norms $\|\mathbf{x}\| /\left\|\mathbf{s}_{0}\right\|$ is used as a normalization factor and $\delta$ is a small dimensionless parameter. Calculating $\mathbf{F x}$ in this manner has several advantages: it uses the forward simulation as a black box, such that no modifications in the code are required. Furthermore, it only requires one forward run for each vector $\mathbf{x}$, such that for example the computation of FA, where $\mathbf{F}$ is an $m \times m$ matrix and $\mathbf{A}$ is an $m \times r$ matrix, has a computational cost equal to $r+1$ calls to forward 


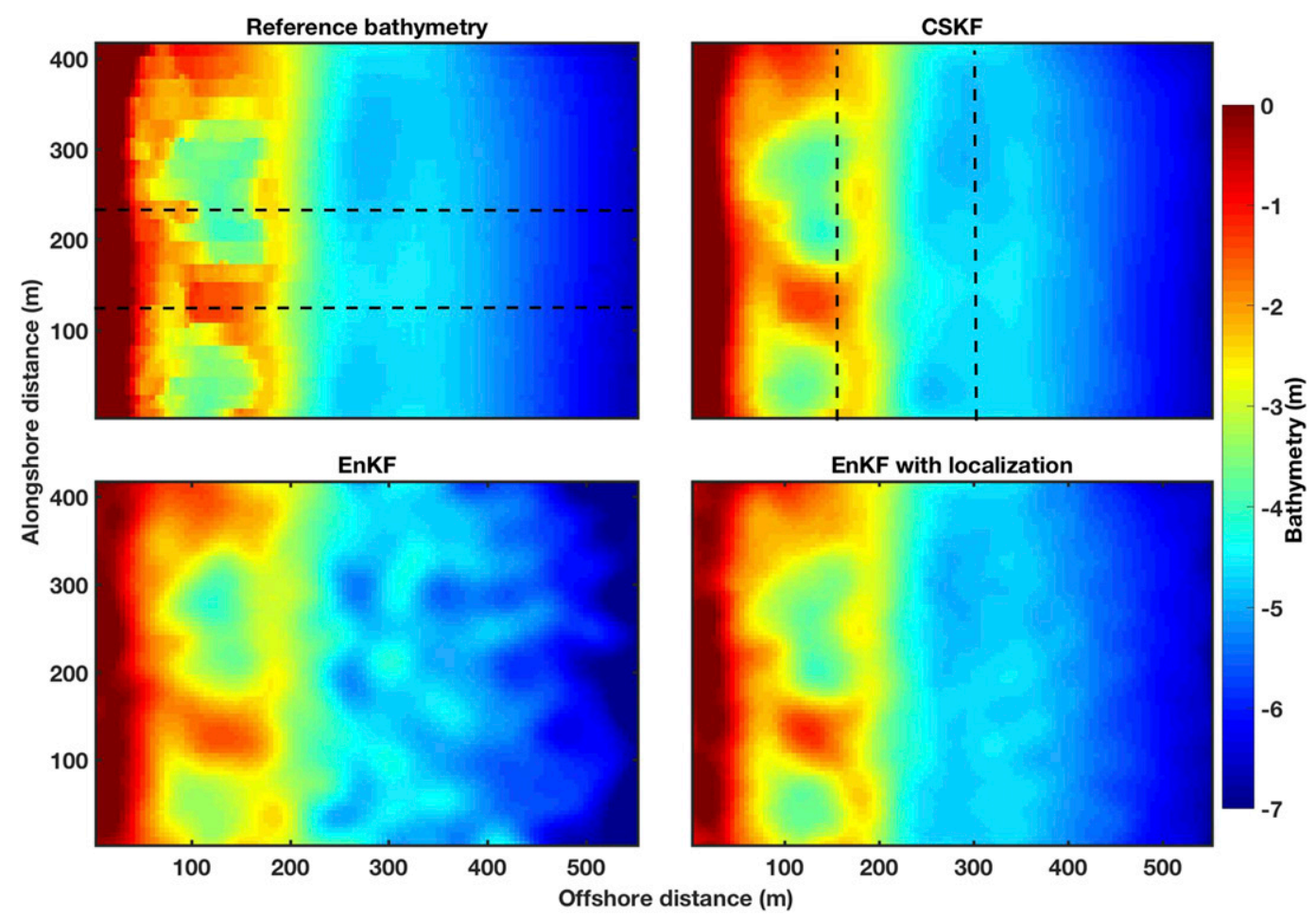

FIG. 1. (top left) Reference bathymetry and (top right) estimated bathymetry by CSKF with rank 100. (bottom left) Estimated bathymetry by the ensemble-based method without localization with ensemble size of 100 and (bottom right) estimated bathymetry by the ensemble-based method with localization with ensemble size of 100 and a localization radius of $60 \mathrm{~m}$. Comparison with the reference bathymetry shows that both the CSKF and localized ensemble-based method provide accurate results, but the results of the ensemble-based method without localization are noisy and inaccurate. The dashed lines on the top-left and top-right panels show the locations of cross-shore and alongshore transects that are used for comparison in Figs. 6 and 7, respectively.

solver (the rank plus 1), whereas the simple computation of the Jacobian matrix $\mathbf{F}$ in the original method requires $m^{2}+1$ evaluation of the forward solver. Finally, these computations can be performed independently and in parallel to further increase computational efficiency.

Although the modifications mentioned above address the computational challenge associated with the large state vector in EKF, computing the inverse of $\left(\mathbf{H}_{t} \mathbf{A}\right) \mathbf{C}_{t+1}^{f}\left(\mathbf{H}_{t} \mathbf{A}\right)^{\mathrm{T}}+\mathbf{R}_{t}$ in Eq. (3c) is computationally expensive for problems with a large number of observations. To overcome this problem, we take advantage of the fact that the rank of $A$ is smaller than the number of observations and use the Sherman-Morrison-Woodbury formula (Sherman and Morrison 1950; Khodayi-mehr et al. 2018a) to compute the inverse of this matrix as below:

$$
\begin{gathered}
{\left[\left(\mathbf{H}_{t} \mathbf{A}\right) \mathbf{C}_{t+1}\left(\mathbf{H}_{t} \mathbf{A}\right)^{\mathrm{T}}+\mathbf{R}_{t}\right]^{-1}=\mathbf{R}_{t}^{-1}-\mathbf{R}_{t}^{-1}\left(\mathbf{H}_{t} \mathbf{A}\right)} \\
\quad \times\left[\mathbf{C}_{t+1}^{-1}+\left(\mathbf{H}_{t} \mathbf{A}\right)^{\mathrm{T}} \mathbf{R}_{t}^{-1}\left(\mathbf{H}_{t} \mathbf{A}\right)\right]^{-1}\left(\mathbf{H}_{t} \mathbf{A}\right)^{\mathrm{T}} \mathbf{R}_{t}^{-1}
\end{gathered}
$$

Usually the observation error covariance matrix $\mathbf{R}$ is diagonal and constant; therefore, $\mathbf{R}^{-1}$ can be computed easily in the beginning and be used for all steps. Also, the matrices $\mathbf{C}_{t+1}^{-1}$ and $\mathbf{C}_{t+1}^{-1}+\left(\mathbf{H}_{t} \mathbf{A}\right)^{\mathrm{T}} \mathbf{R}_{t}^{-1}\left(\mathbf{H}_{t} \mathbf{A}\right)$ are small $r \times r$ matrices.

Using matrix compression, a matrix-free approach, and the Woodbury formula, the CSKF algorithm can be summarized by the following recurrence:

$$
\begin{aligned}
\mathbf{x}_{t+1}^{f}= & f\left(\mathbf{x}_{t}^{a}\right), \\
\mathbf{C}_{t+1}^{f}= & \left(\mathbf{F}_{t} \mathbf{A}\right) \mathbf{C}_{t}^{a}\left(\mathbf{F}_{t} \mathbf{A}\right)^{\mathrm{T}}+\mathbf{D}_{t}, \\
\mathbf{K}_{t+1}= & \mathbf{A} \mathbf{C}_{t+1}^{f}\left(\mathbf{H}_{t} \mathbf{A}\right)^{\mathrm{T}}\left\{\mathbf{R}_{t}^{-1}-\mathbf{R}_{t}^{-1}\left(\mathbf{H}_{t} \mathbf{A}\right)\right. \\
& \left.\times\left[\mathbf{C}_{t+1}^{-f}+\left(\mathbf{H}_{t} \mathbf{A}\right)^{\mathrm{T}} \mathbf{R}_{t}^{-1}\left(\mathbf{H}_{t} \mathbf{A}\right)\right]^{-1}\left(\mathbf{H}_{t} \mathbf{A}\right)^{\mathrm{T}} \mathbf{R}_{t}^{-1}\right\}, \\
\mathbf{x}_{t+1}^{a}= & \mathbf{x}_{t+1}^{f}+\mathbf{K}_{t+1}\left[\mathbf{y}_{t+1}-h\left(\mathbf{x}_{t+1}^{f}\right)\right], \\
\mathbf{C}_{t+1}^{a}= & \mathbf{C}_{t+1}^{f}-\mathbf{A}^{\mathrm{T}} \mathbf{K}_{t+1}\left(\mathbf{H}_{t} \mathbf{A}\right) \mathbf{C}_{t+1}^{f},
\end{aligned}
$$

where $\mathbf{D}_{t}=\mathbf{A}^{\mathrm{T}} \mathbf{A} \mathbf{Q}_{t} \mathbf{A}$.

Table 1 compares the computational cost of the proposed CSKF method and EnKF in terms of the number 
of forward model evaluations and the cost of matrix operations. For the case that the rank used in CSKF is the same as the ensemble size in EnKF, these methods have roughly the same computational cost in terms of the number of forward model evaluations. It should be noted that the computational cost of localized EnKF is roughly the same as EnKF as it has the same number of forward model evaluations. However, in terms of the computational cost of matrix operations, the proposed CSKF method is computationally more efficient than EnKF for the problems with a large number of measurements $(r \ll n)$. Note that the Woodbury formula can be used similarly in the EnKF algorithm to reduce its computational cost.

\section{Applications}

To demonstrate the accuracy and robustness of the CSKF method, we consider twin tests with synthetic observations of wave celerity and wave height, while the bathymetry profiles are chosen based on surveys taken by the U.S. Army Corps of Engineer Field Research Facility (FRF) in Duck, North Carolina. The FRF has been the site of numerous field investigations into wave dynamics and coastal processes (Birkemeier and Thornton 1994). It maintains extensive, accurate survey data and continuously monitored wave and tidal data (http://www.frf.usace.army.mil). The FRF features a relatively straight coastline and is considered representative of many U.S. beaches in terms of wave climate, storm exposure, and sand size (Birkemeier et al. 1985). The beach has a tidal range of $0.5-2.0 \mathrm{~m}$.

The numerical model used for simulation is SteadyState Spectral Wave (STWAVE) (Massey et al. 2011; Smith et al. 2001). STWAVE simulates nearshore wave propagation and transformation including refraction, shoaling, breaking, and wind-wave generation. The STWAVE governing equations are numerically solved using finite-difference methods on a uniform grid with square cells. STWAVE operates in a local coordinate system, with the $x$ axis oriented in the cross-shore direction and the $y$ axis oriented along the shore. STWAVE is used as a robust nearshore wave transformation model that has been developed and maintained by the U.S. Army Engineer Research and Development Center [ERDC; formally known as Waterways Experiment Station (WES)] (Smith et al. 2001; Smith 2001). STWAVE can generate different types of measurements such as wave celerity, wave height, wave direction, and wave period and the CSKF method can be generalized to use several different measurement types. Therefore, STWAVE is used here, as it would be easier to extend our work to use other types of measurements.
TABLE 2. Inversion parameters for the test cases.

\begin{tabular}{|c|c|c|c|}
\hline Parameter & Description & Case 1 & Case 2 \\
\hline \multicolumn{4}{|c|}{ Measurement error } \\
\hline$n_{\text {obs }}$ & Number of measurements & $9130 \times 2$ & 3876 \\
\hline$\sigma_{\text {cel }}$ & $\begin{array}{l}\text { Standard deviation of wave } \\
\text { celerity measurement } \\
\text { error }\left(\mathrm{m} \mathrm{s}^{-1}\right)\end{array}$ & 0.1 & 0.1 \\
\hline$\sigma_{\mathrm{wh}}$ & $\begin{array}{l}\text { Standard deviation of wave } \\
\text { height measurement } \\
\text { error }(\mathrm{m})\end{array}$ & 0.2 & - \\
\hline \multicolumn{4}{|c|}{ Inversion parameters } \\
\hline$m$ & Number of unknowns & 9130 & 3876 \\
\hline$\sigma_{h}^{2}$ & Prior variance $\left(\mathrm{m}^{2}\right)$ & 1 & 1 \\
\hline$L_{x}^{n}, L_{y}$ & Scale parameter $(\mathrm{m})$ & 60,45 & 100,80 \\
\hline
\end{tabular}

\section{Problem formulation and measurements}

In the twin synthetic problems, the true bathymetry profiles are assumed to be unknown and the prior (meaning the assumed initial values at time " 0 ," before observations are assimilated) bathymetry profiles are assumed to increase linearly with the offshore distance. Synthetic measurements (wave celerity and wave height measurements for the first test case and wave celerity measurements for the second test case) are generated by running STWAVE with the true bathymetry profiles and then contaminated with white Gaussian noise for each data assimilation step, to test the robustness of the estimation approach to unavoidable observation and modeling errors. Since different measurement types can have different accuracy, the magnitude of white Gaussian noise added to generated wave height is different from the magnitude of white Gaussian noise added to generate wave celerity in the first test case.

For bathymetry application, we use a random walk forecast model shown in Eq. (9) which is used because of the absence of a good model to approximate the true evolution of the bathymetry profile and high rate of data acquisition. This essentially means the only source of information we have to estimate bathymetry profile are its measurements, which are related to bathymetry as shown in Eq. (2). Also, for this particular application, the matrix $\mathbf{F}$ in CSKF algorithm should be replaced with identity matrix I because we use random walk forecast model:

$$
\mathbf{x}_{t+1}=\mathbf{x}_{t}+\mathbf{w}_{t} .
$$

Therefore, the CSKF algorithm updates the estimated bathymetry as

$$
\hat{\mathbf{x}}_{t+1 \mid t+1}=\hat{\mathbf{x}}_{t \mid t}+\mathbf{K}_{t+1}\left[\mathbf{y}_{t+1}-h\left(\hat{\mathbf{x}}_{t \mid t}\right)\right],
$$

where the variable $y$ represents wave celerity measurements. The nonlinear STWAVE function shown as 

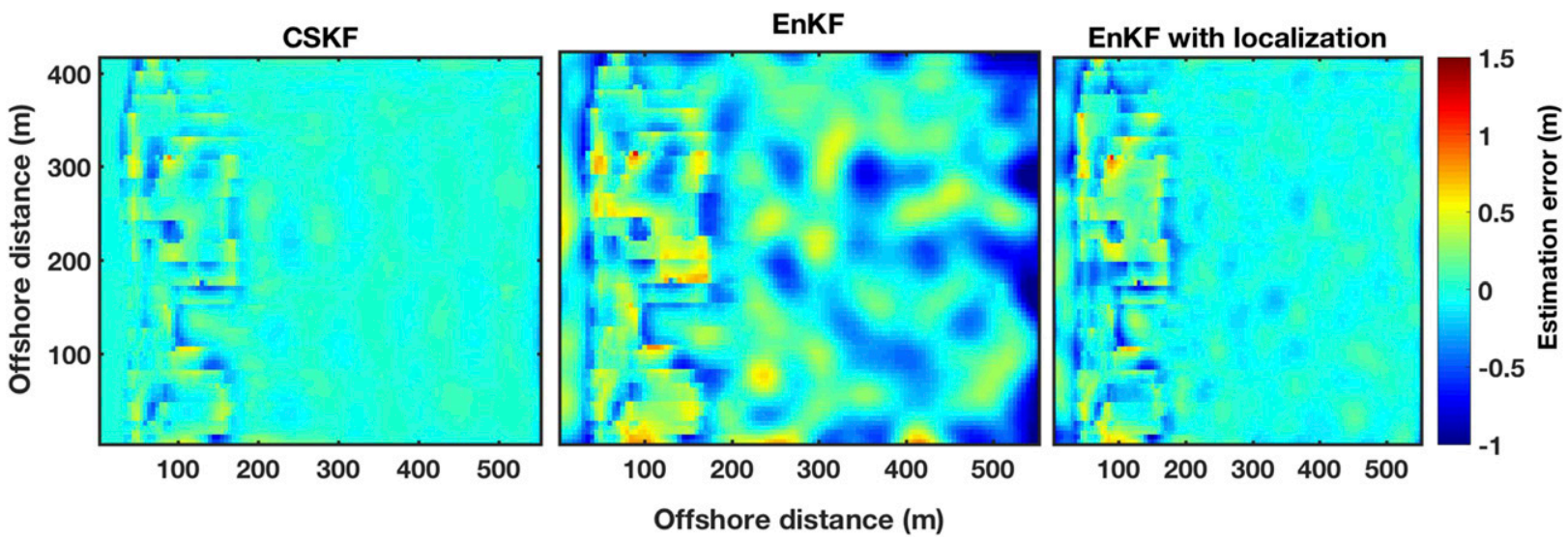

FIG. 2. Comparison of the bathymetry estimation error by (left) CSKF, (center) ensemble-based method without localization, and (right) ensemble-based method with localization. The comparison shows that both the CSKF and ensemble-based method with localization are more accurate than ensemble-based method without localization. However, CSKF estimation error is smaller, especially in smaller offshore distances. This can be better shown by comparison of the RMSE, where RMSE of estimation results from left to right are $0.092,0.293$, and $0.133 \mathrm{~m}$.

$h(\cdots)$ relates the ocean surface observations to bathymetry.

In addition to the comparison to the true bathymetry for validation of CSKF, we use this problem to conduct an error analysis of CSKF in comparison with two ensemble-based methods, one with covariance localization and one without covariance localization, similar to (Wilson et al. 2014, 2010). In the ensemble-based

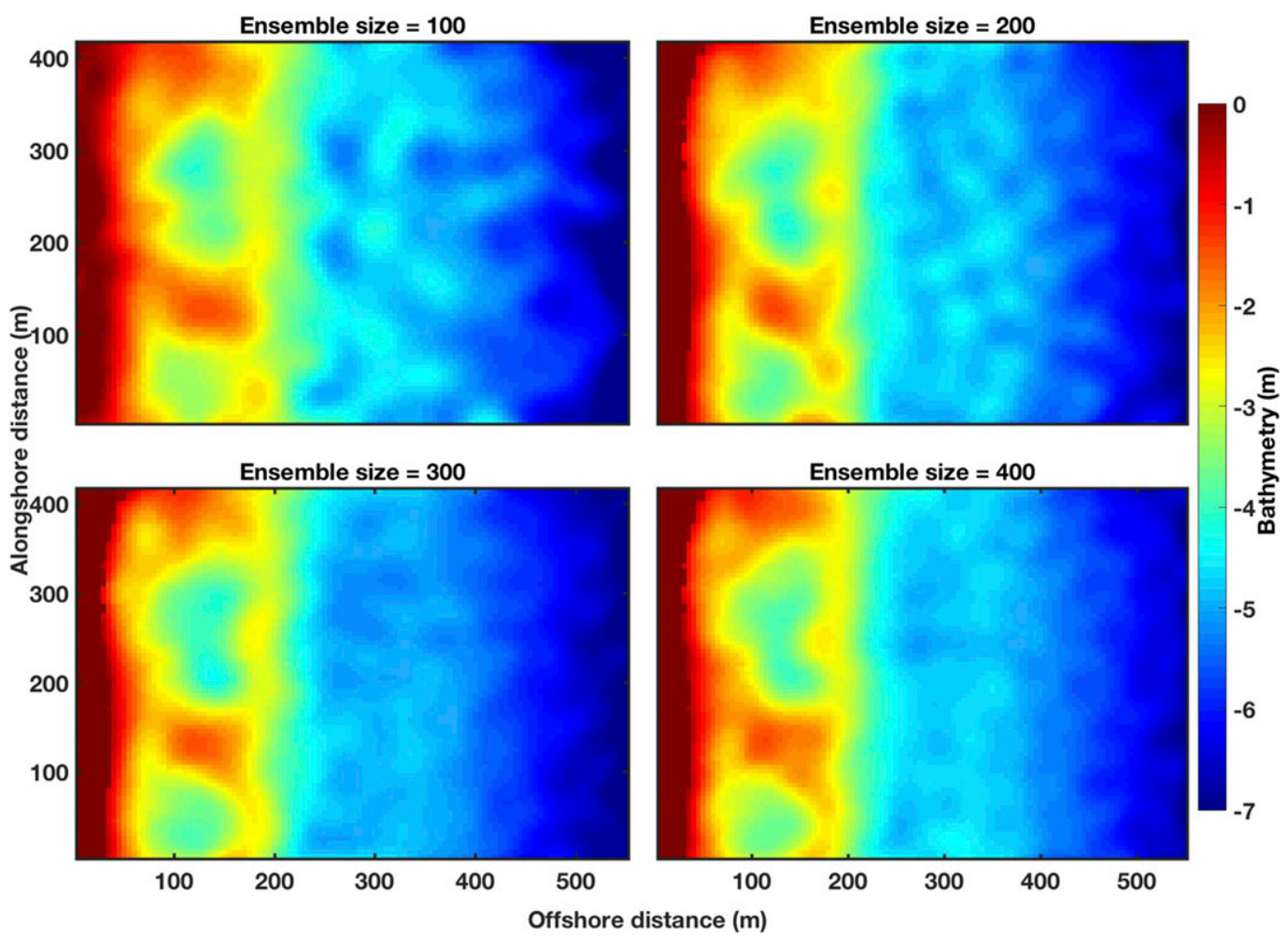

FIG. 3. Comparison of the bathymetry estimation results for the ensemble-based method without localization with different numbers of ensembles. The accuracy of the results increases as the ensemble size increases; however, the computational cost of the method increases as well. The RMSE values for ensemble size (top left) 100, (top right) 200, (bottom left) 300, and (bottom right) 400 are $0.29,0.16,0.14$, and $0.13 \mathrm{~m}$, respectively. 


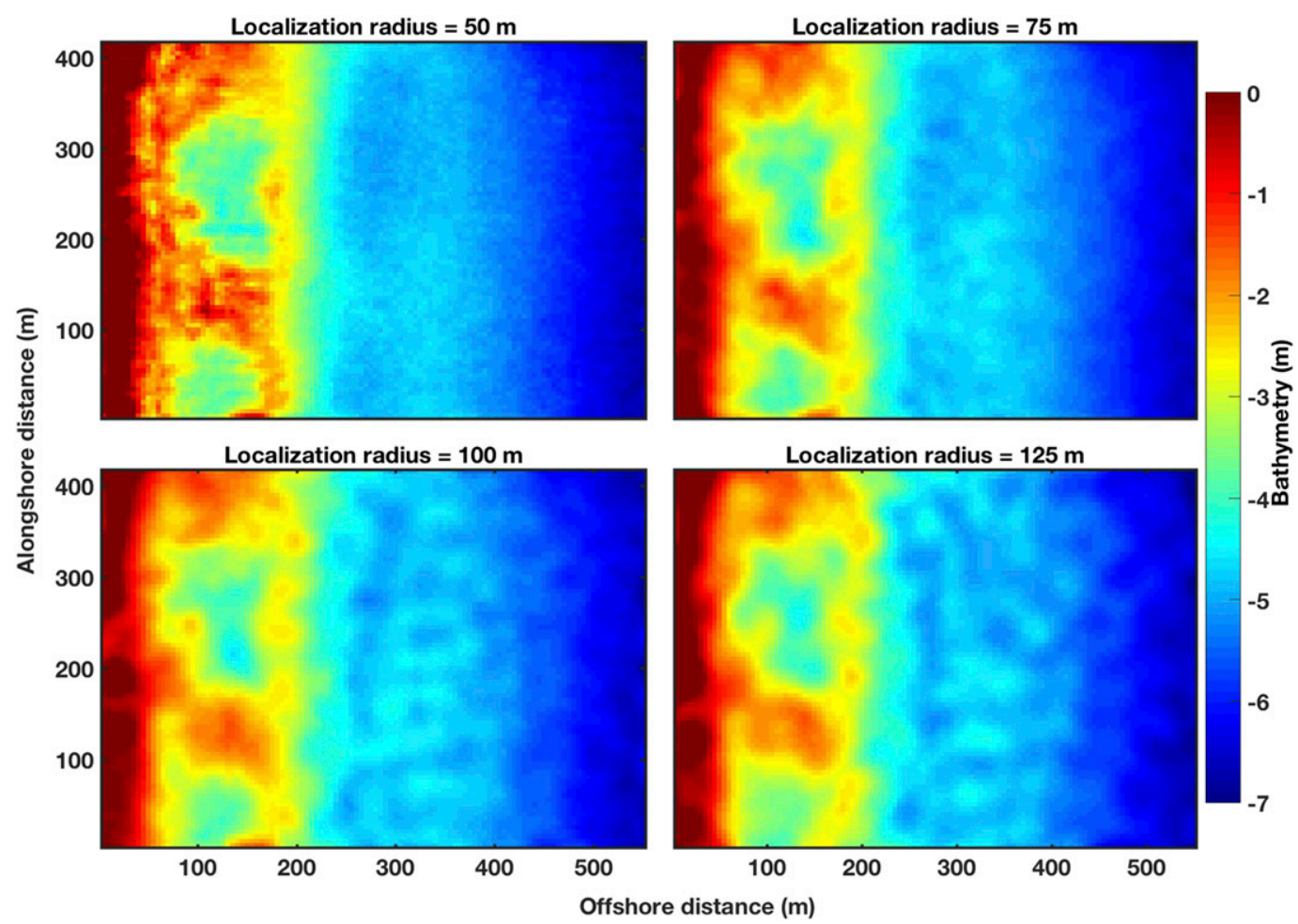

FIG. 4. Comparison of the bathymetry estimation by the ensemble-based method with different localization radius. The comparison shows that localized ensemble-based method accuracy highly depends on the localization radius. (bottom right) For a radius of $125 \mathrm{~m}$, the results show small-scale variabilities that do not exist in the reference bathymetry.

methods, the bathymetry profile is updated at each data assimilation step as

$$
\begin{aligned}
& \hat{\mathbf{x}}_{t+1 \mid t+1_{i}}=\mathbf{x}_{t \mid t_{i}}+\mathbf{P}_{t} \mathbf{H}^{\mathrm{T}}\left(\mathbf{H} \mathbf{P}_{t} \mathbf{H}^{\mathrm{T}}+\mathbf{R}\right)^{-1}\left[\tilde{\mathbf{y}}_{t+1_{i}}-h\left(\hat{\mathbf{x}}_{t \mid t_{i}}\right)\right], \\
& \hat{\mathbf{x}}_{t+1 \mid t+1}=\frac{1}{N_{\text {ens }}} \sum_{i=1}^{N_{\text {ens }}} \hat{\mathbf{x}}_{t+1 \mid t+1_{i}}
\end{aligned}
$$

where $\tilde{\mathbf{y}}_{t+1} \sim N\left(\mathbf{y}_{t+1}, \mathbf{R}\right)$ and the $\mathbf{P}_{t} \mathbf{H}^{\mathrm{T}}$ and $\mathbf{H P}_{t} \mathbf{H}^{\mathrm{T}}$ terms are computed as

$$
\mathbf{P}_{t} \mathbf{H}^{\mathrm{T}}=\frac{1}{N_{\mathrm{ens}}-1} \sum_{i=1}^{N_{\text {ens }}}\left(\mathbf{x}_{t \mid t_{i}}-\overline{\mathbf{x}_{t \mid t}}\right)\left[h\left(\mathbf{x}_{t \mid t_{i}}\right)-\overline{h\left(\mathbf{x}_{t \mid t}\right)}\right]^{\mathrm{T}},
$$

$\mathbf{H} \mathbf{P}_{t} \mathbf{H}^{\mathrm{T}}=\frac{1}{N_{\mathrm{ens}}-1} \sum_{i=1}^{N_{\text {ens }}}\left[h\left(\mathbf{x}_{t \mid t_{i}}\right)-\overline{h\left(\mathbf{x}_{t \mid t}\right)}\right]\left[h\left(\mathbf{x}_{t \mid t_{i}}\right)-\overline{h\left(\mathbf{x}_{t \mid t}\right)}\right]^{\mathrm{T}}$,

where $\quad \overline{\mathbf{x}_{t \mid t}}=\left(1 / N_{\mathrm{ens}}\right) \sum_{i=1}^{N_{\mathrm{ens}}} \mathbf{x}_{t \mid t_{i}} \quad$ and $\quad \overline{h\left(\mathbf{x}_{t \mid t}\right)}=$ $\left(1 / N_{\mathrm{ens}}\right) \sum_{i=1}^{N_{\mathrm{ens}}} h\left(\mathbf{x}_{t \mid t_{i}}\right)$.

Although the ensemble-based methods are computationally efficient, they usually suffer from spurious model correlation, ensemble spread reduction, and ensemble collapse. Also, for data-intensive problems, these methods require a large ensemble to provide a good accuracy, which increases the computational cost. To tackle these issues partially, we implement a covariance localization strategy using a compactly supported piecewise rational fifth-order polynomial function following Wilson et al. (2014).

\section{Results and discussion}

\section{a. The first test problem}

The first test case we consider is a steady-state bathymetry estimation problem with a bathymetry profile taken from an FRF survey for 26 July 2016 (Fig. 1) and represents nearshore profiles typical of calm summer periods. The survey data were projected to the rectangular computational grid using bilinear interpolation. Also, bathymetry values are not constrained to be negative and it is physically acceptable to have positive bathymetry in the domain especially near the shore and they represent dry land. However, positive values are improper when they are given as input values to the numerical model, STWAVE, because the model cannot 


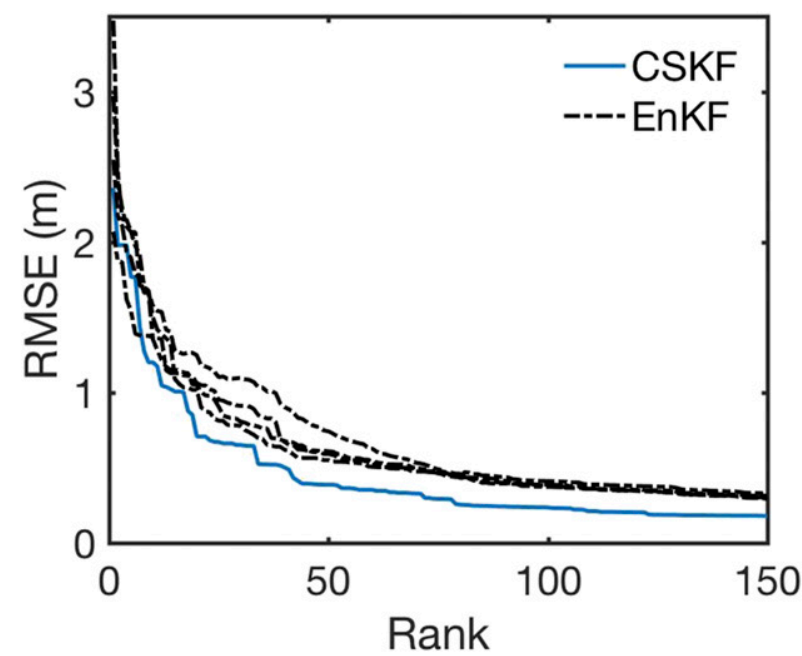

FIG. 5. RMSE between the reference bathymetry and its projection to eigenspace of the prior covariance vs the number of rank for CSKF and the ensemble-based method. We used different random seed numbers to generate the realizations.

generate the corresponding wave celerity and wave height observations for the positive values. Therefore, similar to Wilson et al. (2014), we enforce bathymetry to be negative by truncating the positive values of bathymetry to a minimum value of $-0.05 \mathrm{~m}$. Then synthetic wave celerity and wave height observations are generated by running STWAVE with this bathymetry profile and then adding white Gaussian noise with standard deviations of $0.1 \mathrm{~m} \mathrm{~s}^{-1}$ and $0.2 \mathrm{~m}$ to the generated wave celerity and wave height measurements, respectively.

The computational domain for this problem is a $550-\mathrm{m}$ across-shore and 415-m alongshore area south of the FRF pier which is a subsample of the full FRF survey, and it is divided into a $110 \times 83$ grid. The boundary conditions for the simulations are taken from the FRF 8 - $\mathrm{m}$ array. The FRF 8 - $\mathrm{m}$ array is located $1 \mathrm{~km}$ offshore at an approximate depth of $8 \mathrm{~m}$. It consists of nine alongshore and six across-shore near-bottom pressure sensors (Hathaway and Long 2008). Offshore wave conditions for the first test problem were taken on 22 July 2016, which was a relatively calm period with wave heights $\leq 1 \mathrm{~m}$ and peak periods between 8 and 10s sufficiently close to the survey date of 26 July.

In this test case, the accuracy of CSKF is compared with that of two ensemble-based methods with the same computational cost. All filters are initiated with the same initial condition and the same set of noisy measurements. Also, the methods use a Gaussian kernel $\mathbf{Q}_{i, j}=\sigma_{h}^{2} \exp \left\{-\left[\left(x_{i}-x_{j}\right) / L_{x}\right]^{2}-\left[\left(y_{i}-y_{j}\right) / L_{y}\right]^{2}\right\}$ and
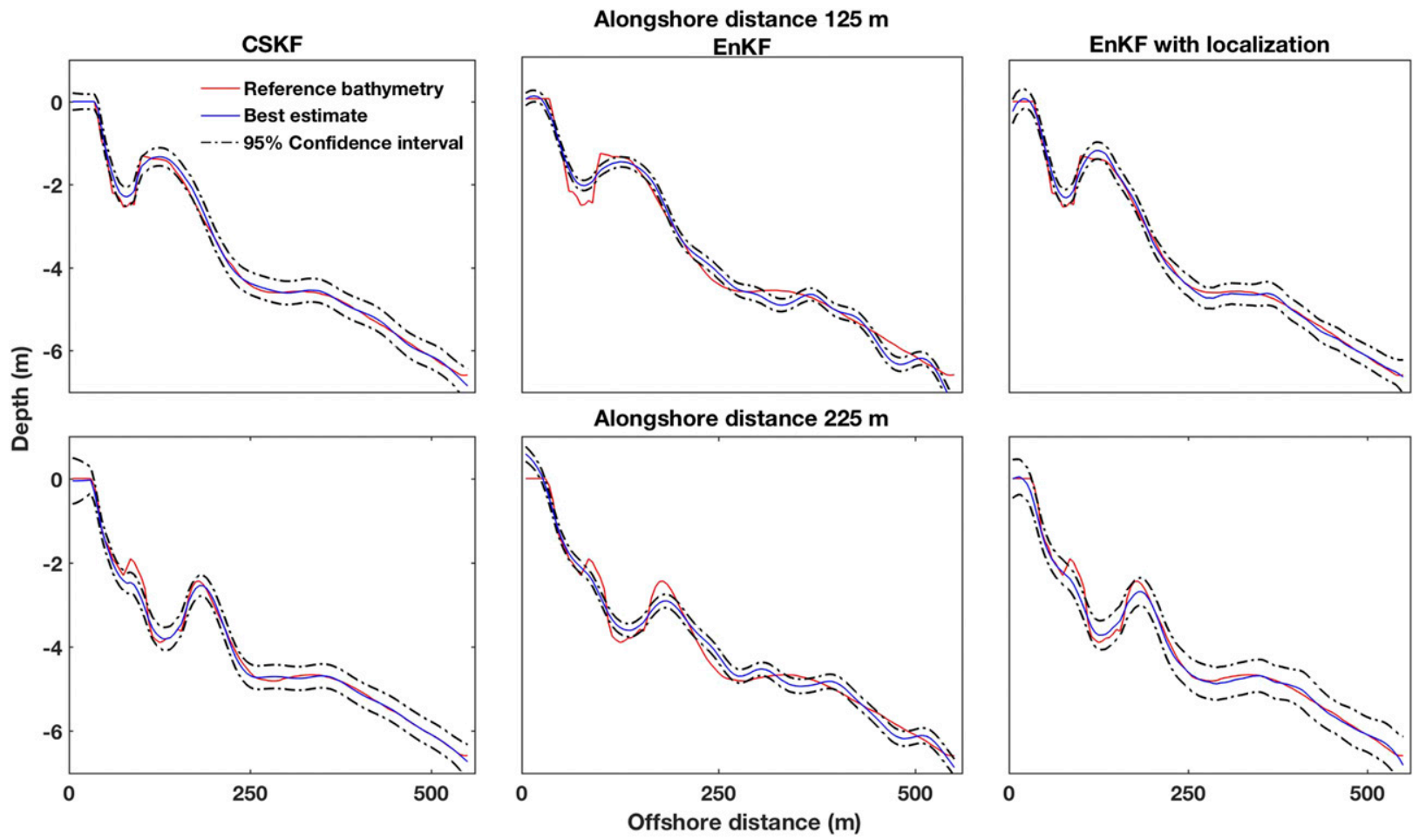

FIG. 6. Estimated bathymetry and its estimated uncertainty for two cross-shore transects, with alongshore distances of (top) 125 and (bottom) $225 \mathrm{~m}$, obtained by using (left) CSKF with rank 100 and (center),(right) ensemble-based methods with an ensemble size of 100 . The results indicate that the ensemble-based method without localization experiences ensemble collapse (underestimation of uncertainty). The localization technique alleviates this issue; however, the mean estimate of the localized ensemble-based method is not as accurate as the CSKF estimated mean. 

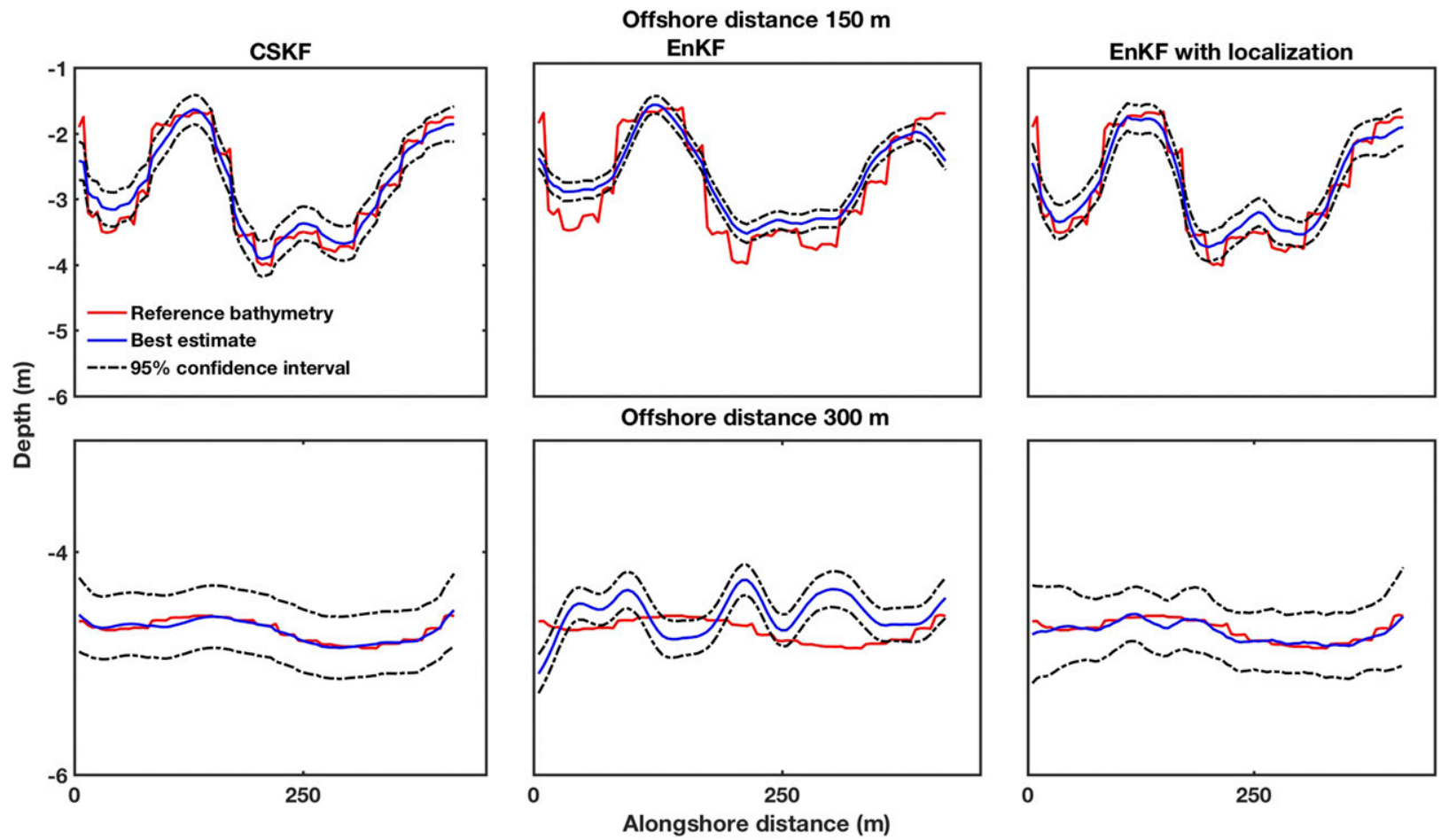

FIG. 7. Estimated bathymetry and its estimated uncertainty for two alongshore transects, with offshore distances of (top) 150 and (bottom) $300 \mathrm{~m}$, obtained by using (left) CSKF with rank 100 and (center),(right) ensemble-based methods with an ensemble size of 100. The results indicate that the ensemble-based method without localization cannot provide a good estimate for bathymetry especially for large offshore distances and also it experiences ensemble collapse (underestimation of uncertainty).

the observation error covariance matrix $\mathbf{R}$ is given as below:

$$
\mathbf{R}=\left[\begin{array}{cc}
\sigma_{\mathrm{cel}}^{2} \mathbf{I}_{n c \times n c} & 0 \\
0 & \sigma_{\mathrm{wh}}^{2} \mathbf{I}_{n h \times n h}
\end{array}\right],
$$

where $\mathbf{I}_{n c \times n c}$ and $\mathbf{I}_{n h \times n h}$ are identity matrices and $n c=n h=0.5 n_{\mathrm{obs}}$. The inversion parameters are listed in Table 2.

Figure 1 demonstrates the reference bathymetry profile and the estimated bathymetry by CSKF with rank 100 and ensemble-based methods with ensemble size 100 . Comparison with the reference bathymetry shows that CSKF and localized ensemble-based method provide accurate results but the results of the ensemble-based method without localization are noisy and less accurate. The performance comparison of these methods is better demonstrated in Fig. 2 where the estimation errors are shown in the domain. As this figure demonstrates, CSKF captures the small-scale features near the shore, offshore distances between 50 and $200 \mathrm{~m}$, better than the localized ensemble-based method. The RMSE values for the estimation results of CSKF, localized ensemble-based method, and ensemble-based method without localization are respectively $0.092,0.133$, and $0.293 \mathrm{~m}$, which shows that CSKF is more accurate than both ensemblebased techniques.

Figure 3 shows the effect of ensemble size on the filter's performance. The accuracy of the ensemble-based method without localization results increases as the

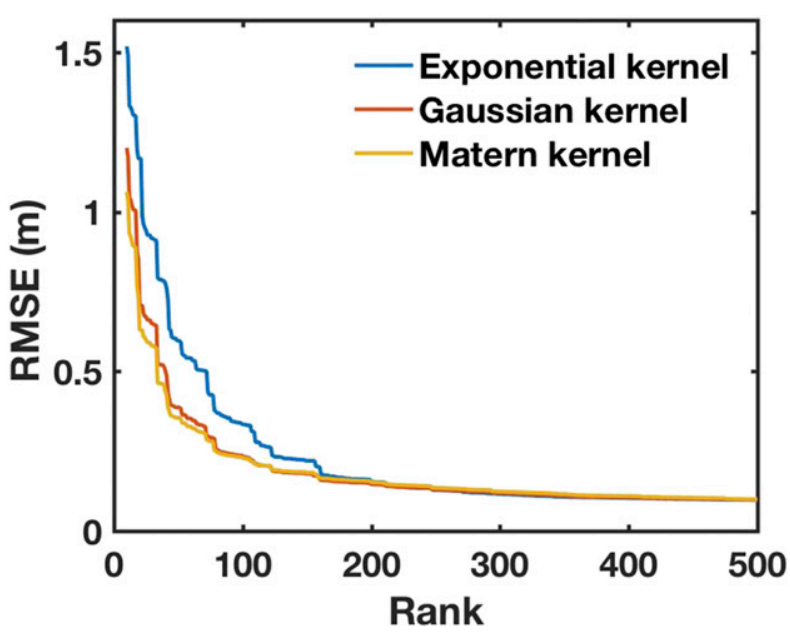

FIG. 8. RMSE values between the true and projected bathymetry profiles onto the eigenspace of the prior covariance vs the number of eigenvectors. 

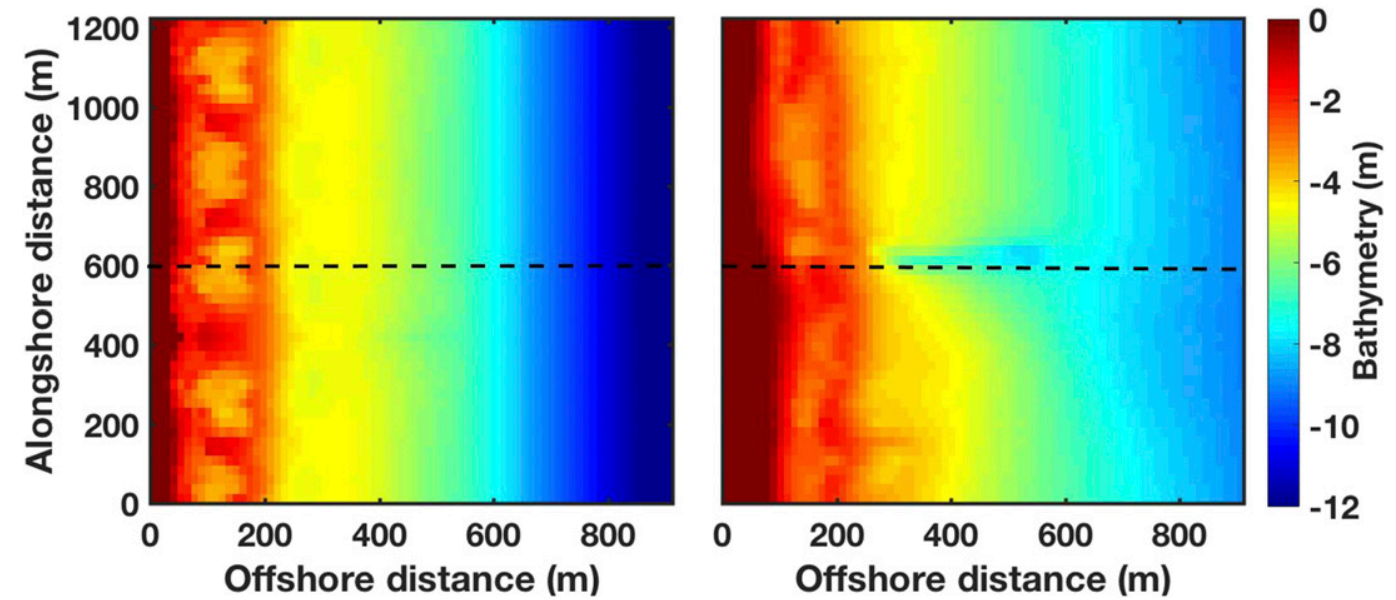

FIG. 9. Reference bathymetry profiles used to generate wave celerity measurement for the second test problem (The positive values for bathymetry are projected to $-0.05 \mathrm{~m}$, because the input bathymetry values for the forward model should be nonpositive). The bathymetry profile for the second test case varies linearly from (left) bathymetry profile A shown to (right) bathymetry profile B in five time steps. The dashed line shows the location of cross-shore transect that is used for comparison in Fig. 13.

ensemble size increases; however, the computational cost of the method increases as well while CSKF can produce more accurate results with less computational cost. For example, the computational cost of the ensemble-based method with 400 ensemble realizations is 4 times greater than the computational cost of CSKF with rank 100 while the RMSE of CSKF estimation is less than that of the ensemble-based method (i.e., $0.092<0.133 \mathrm{~m}$ ).

We next investigate the effect of localization radius parameter on the estimation accuracy of the localized ensemble-based method. As expected, when the localization radius is small, the estimation is more accurate with small-scale noise in the results (Fig. 4). The smallscale random artifacts in the estimates are due to the use of a small localization radius parameter to obtain higher estimation accuracy (Tsyrulnikov 2009). As the localization radius increases, the estimation accuracy decreases and the results converge to that of the ensemble-based method without localization. Therefore, to achieve the desired accuracy, one needs to increase the ensemble size, which increases the computational cost of the method. These results indicate that the localization parameter greatly affects the performance of the filter. This is one of the weaknesses of this method since to the authors' knowledge there is no specific way to determine this parameter and this is usually done in a heuristic way.

Figure 5 demonstrates the variation of RMSE value between the reference bathymetry and its projection to eigenspace of the prior covariance versus the rank for CSKF and the ensemble size for the ensemble-based method without localization with four different random seed numbers. The covariance matrix for the ensemble-based method can be described with the empirical covariance matrix sampled from the actual covariance matrix. These RMSE values represent the minimum error that CSKF and ensemble-based methods can achieve under ideal conditions, for example, a good choice of the prior, the full-rank sensitivity matrix, and small measurement error. The results show that RMSE values for the CSKF method are generally smaller than those obtained from the ensemble-based method, because in the ensemble-based methods the actual rank of the ensemble covariance matrix might be much smaller than the ensemble size when the ensemble size is not large due to intrasample multicollinearity introduced by the pseudorandom sampler and numerical error.

Figure 6 depicts the a posteriori bathymetry mean with $95 \%$ credible intervals (also known as Bayesian confidence intervals) for two cross-shore transects at $y=125$ and $y=225 \mathrm{~m}$. The CSKF method captures the bathymetry profile surprisingly well, though the prior bathymetry mean varied only linearly with the offshore distance and did not provide any information regarding bathymetry features between 50 - and 200-m offshore distances. While the localized version of the ensemblebased method can capture most of the variability in the bathymetry profile, the ensemble-based method without localization experiences ensemble collapse (underestimation of uncertainty) and could not capture the bathymetry profile in large offshore distances. This is better shown in Fig. 7 where the bathymetry mean with its $95 \%$ credible intervals for two alongshore transects at $x=150$ and $x=300 \mathrm{~m}$ are plotted for each method.

Next, we perform a projection analysis on the true bathymetry images using three different covariance 


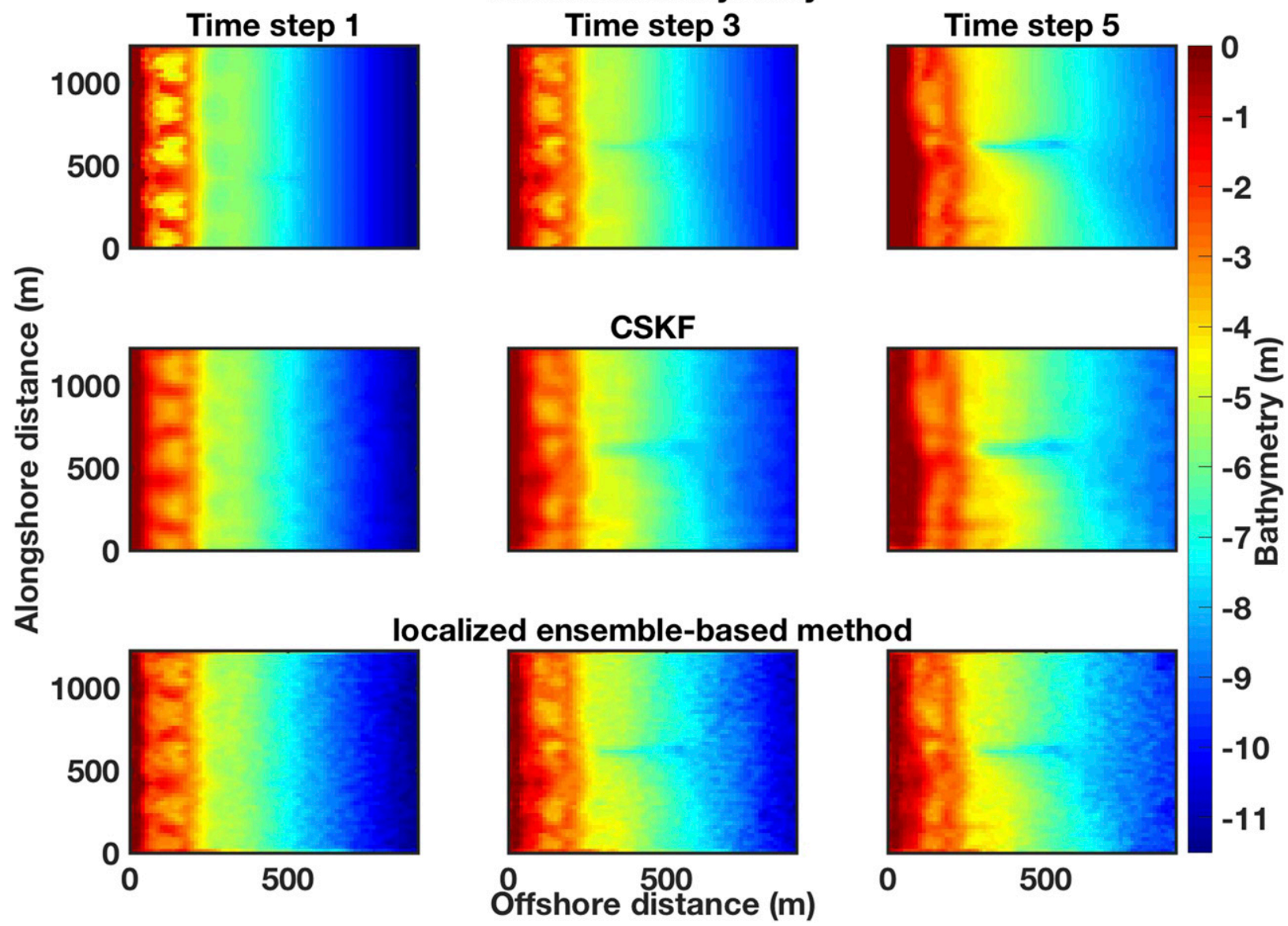

FIG. 10. Comparison at three time steps of (top) reference bathymetry, (middle) CSKF estimation for bathymetry, and (bottom) localized ensemble-based method estimation for bathymetry. The results show that CSKF estimates evolving bathymetry robustly.

kernels to validate the choice of the kernel function and the number of bases used to describe the reference field accurately and efficiently. For this, we project the reference bathymetry image onto the subspace spanned by the covariance matrix and check how the projected image is different from the true one. In Fig. 8, the RMSE values between the true and reconstructed images are plotted against the number of eigenvectors/ principal components used for the reconstruction using these different kernel functions with same length scales ( $L_{x}=60 \mathrm{~m}$ and $L_{y}=45 \mathrm{~m}$ for $x$ and $y$ directions, respectively). The results show that the Gaussian kernel and the Matern kernel can capture the reference bathymetry profile better than the exponential kernel and the RMSE value for this kernel almost converges to its minimum value with 100 bases.

\section{b. The second test problem}

The second test case is a bathymetry estimation problem for a temporally evolving bathymetry where the bathymetry varies from profile A (Fig. 9, left) to profile B (Fig. 9, right) in five data assimilation steps and reflects the stronger wave climate and effects typical of winter conditions at the FRF. Profile A is obtained by extending and repeating the bathymetry profile from the first test case in offshore and alongshore directions, respectively. While not a realistic physical representation of this system, in order to test the robustness of the algorithm for problems with large temporal variations in the bathymetry, the magnitude of the bathymetry in profile A was scaled by a factor of 1.2. This scaling increases the magnitude of the bathymetry variation at each time step. Profile B is taken from FRF survey for January 2016. As in the first test case, the survey data are projected to the rectangular computational grid using bilinear interpolation. Similar to the first test case, we enforce bathymetry to be negative by truncating the positive values of bathymetry to a minimum value of $-0.05 \mathrm{~m}$. Also, the synthetic wave celerity observations are generated by running STWAVE with 


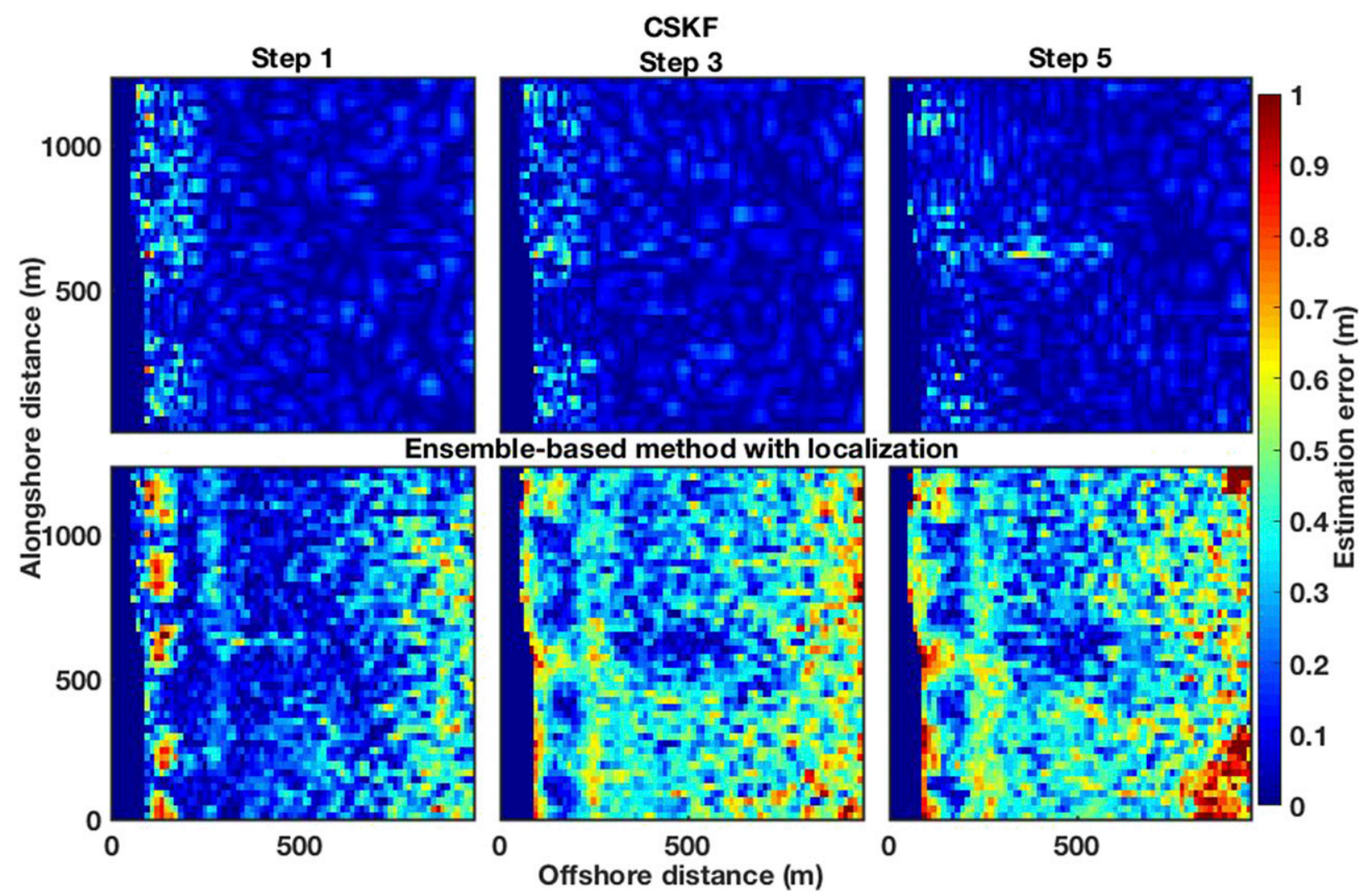

FIG. 11. Comparison of the magnitude of bathymetry estimation error by (top) CSKF with RMSE values of (left) 0.11 , (center) 0.11 , and (right) $0.13 \mathrm{~m}$ and (bottom) the localized ensemble-based method with RMSE values of (left) 0.24, (center) 0.26 , and (right) $0.38 \mathrm{~m}$. The comparison shows that CSKF captures small features better than the localized ensemble-based method. Also, the absolute error of CSKF estimation is smaller than the ensemble-based method estimation in most of the domain.

this bathymetry profile and then adding white Gaussian noise with standard deviation $=0.1 \mathrm{~m} \mathrm{~s}^{-1}$. The computational domain for this problem is a 912-m acrossshore and 1224-m alongshore area south of the FRF pier which is divided into a $76 \times 51$ grid.

The performance of CSKF is compared with that of the localized ensemble-based method (Wilson et al. 2014) with the same computational cost. Both filters use the same Gaussian kernel $\mathbf{Q}_{i, j}=\sigma_{h}^{2} \exp \left\{-\left[\left(x_{i}-x_{j}\right) / L_{x}\right]^{2}-\right.$ $\left.\left[\left(y_{i}-y_{j}\right) / L_{y}\right]^{2}\right\}$ and the same observation error covariance matrix $\mathbf{R}=\sigma_{\mathrm{obs}}^{2} \mathbf{I}_{n \times n}$, where $\mathbf{I}_{n \times n}$ is an identity matrix. The inversion parameters are given in Table 2. Figure 10 compares the reference bathymetry with the estimates obtained by CSKF with rank 100 and the localized ensemble-based method with ensemble size $=100$ and localization radius $=90 \mathrm{~m}$ for three different time steps. Both methods provide accurate estimates after the first data assimilation step. However, the localized ensemble-based method overestimates the bathymetry for offshore distance larger than $600 \mathrm{~m}$ in the third and fifth step while CSKF accurately estimates the bathymetry. Figure 11 shows the estimation error indicating that the CSKF estimation error is very small and close to zero in most of the domain except in estimating some small-scale features. However, the localized ensemble-based method estimation error is around $0.5 \mathrm{~m}$ in most of the domain and even $1 \mathrm{~m}$ in some regions. The comparison of the RMSE values for these methods confirms the superior performance of CSKF compared to the localized ensemble-based method. This indicates that CSKF can detect evolving bathymetry more accurately, hence would be more suitable for temporally variable bathymetry identification.

In Fig. 12, the wave celerity estimated by CSKF and the localized ensemble-based method is plotted versus the measured wave celerity at time step 3. CSKF reproduced the wave celerity accurately with RMSE close to the measurement error level of $0.1 \mathrm{~m} \mathrm{~s}^{-1}$. However, the localized ensemble-based method overestimates wave celerity magnitudes, especially for the values less than $4 \mathrm{~m} \mathrm{~s}^{-1}$.

Figure 13 compares the estimated bathymetry by CSKF and the localized ensemble-based method for 


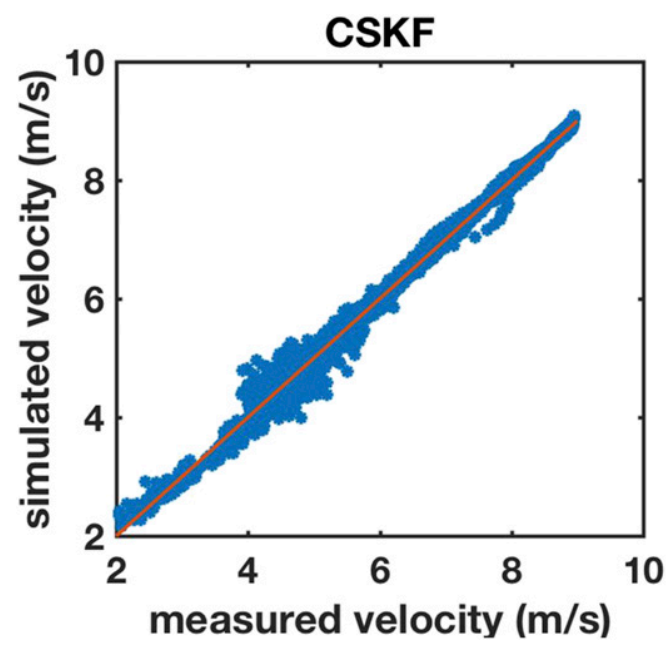

\section{Localized ensemble-based method}

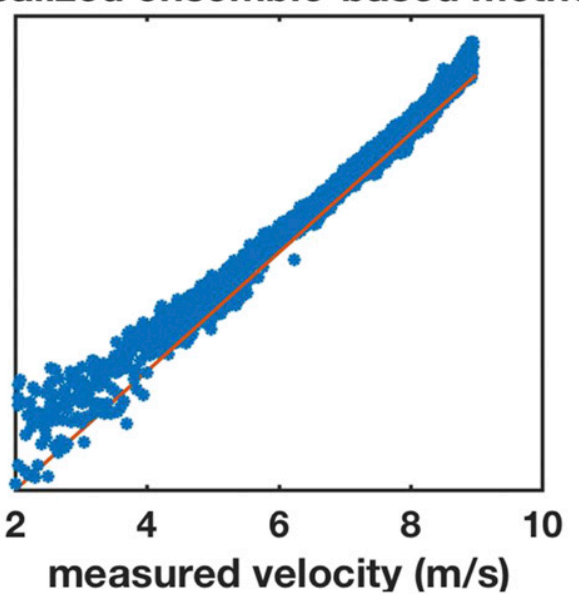

FIG. 12. Observed vs simulated data obtained from (left) CSKF and (right) the localized ensemble-based method. Observation RMSE values for CSKF and the localized ensemble-based method estimations are 0.12 and $0.20 \mathrm{~m} \mathrm{~s}^{-1}$, respectively.

the cross-shore transect at $y=600 \mathrm{~m}$ shown in Fig. 9 . The results show that CSKF estimates the bathymetry accurately even for the region with large spatial variation in bathymetry profile. However, the localized ensemble-based method does not estimate bathymetry as accurately as the CSKF method and it fails to capture the temporal variation of the bathymetry profile.
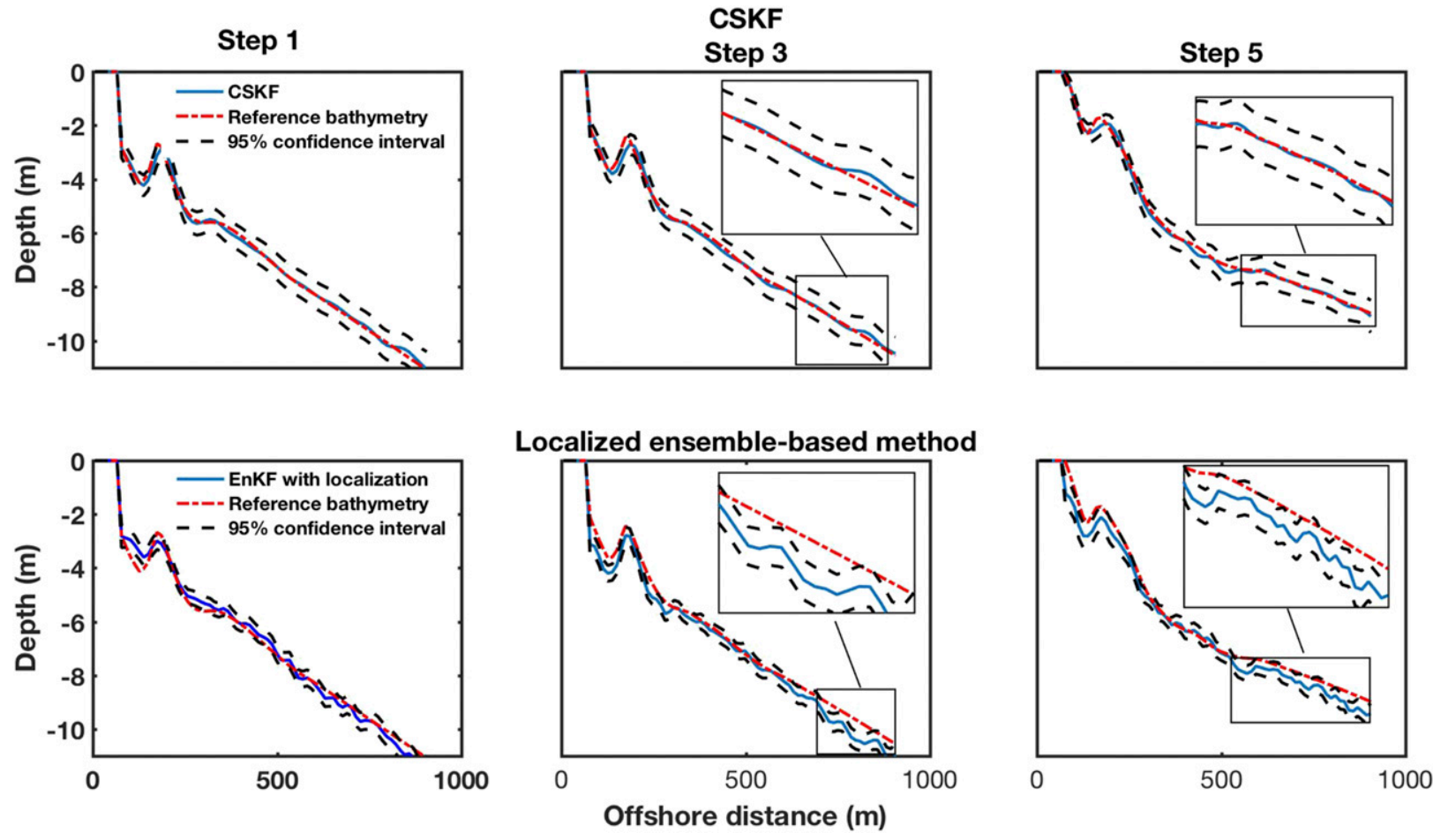

FIG. 13. Estimated bathymetry for a cross-shore transect at an alongshore distance of $600 \mathrm{~m}$ obtained by using (top) CSKF and (bottom) the localized ensemble-based method. CSKF estimates bathymetry accurately while the localized ensemble-based method fails to capture the bathymetry for offshore distances between $\sim 50$ and $\sim 150 \mathrm{~m}$. Also, the localized ensemble-based method overestimates the bathymetry in offshore distances larger than $500 \mathrm{~m}$. 


\section{Conclusions}

This paper presents the application of the compressedstate Kalman filter (CSKF), a fast Kalman filter variant, to coastal bathymetry estimation problems with a large number of unknowns and measurements. The computational speedup of the modified CSKF is achieved by using a low-rank representation for the covariance matrix combined with a matrix-free approach for derivative computations. Here, we include a modification of CSKF using the Woodbury matrix identity that makes it feasible to do state estimation of systems with both large number of unknowns and measurements.

To evaluate the performance of our method, the modified CSKF was applied to two synthetic test problems. In the first one, CSKF estimated the bathymetry and provided corresponding uncertainty accurately for a steady-state bathymetry estimation problem. In the second problem, we investigated the accuracy and robustness of CSKF for a temporally evolving bathymetry. Comparison with two ensemble-based techniques showed that CSKF provides higher accuracy with similar or even smaller computational cost. In addition to being computationally more efficient, CSKF was advantageous because it was more robust in the presence of strong temporal dynamics and it does not require tuning of hyperparameters such as localization radius in the localized ensemble-based method.

The modified CSKF method is a promising algorithm for large-scale data-intensive bathymetry problems. Future work will evaluate the CSKF method on fieldscale problems with observations taken from actual field collections and investigate the effects of nonlinearity on the method's performance.

Acknowledgments. Hojat Ghorbanidehno and Jonghyun Lee were supported in part by an appointment to the Postgraduate Research Participation Program at the U.S. Army Engineer Research and Development Center, Coastal and Hydraulics Laboratory (ERDC-CHL), administered by the Oak Ridge Institute for Science and Education through an interagency agreement between the U.S. Department of Energy and ERDC. Hojat Ghorbanidehno is also supported in part by funding from the Army High Performance Computing Research Center (AHPCRC), sponsored by the U.S. Army Research Laboratory under Contract W911NF-07-2-0027, at Stanford. Jonghyun Lee was supported in part by Hawai' $\mathrm{i}$ Experimental Program to Stimulate Competitive Research (EPSCoR) provided by the National Science Foundation Research Infrastructure Improvement (RII) Track-1: 'Ike Wai: Securing Hawai'i's Water Future Award OIA-1557349. The financial support is gratefully acknowledged. The data for this paper are available upon request to the corresponding author Hojat Ghorbanidehno (hojjatgh@stanford.edu).

\section{REFERENCES}

Aarninkhof, S. G. J., B. G. Ruessink, and J. A. Roelvink, 2005: Nearshore subtidal bathymetry from time-exposure video images. J. Geophys. Res., 110, C06011, https://doi.org/10.1029/ 2004JC002791.

Ahmed, N., T. Natarajan, and K. R. Rao, 1974: Discrete cosine transform. IEEE Trans. Comput., C23, 90-93, https://doi.org/ 10.1109/T-C.1974.223784.

Anderson, B. D., and J. B. Moore, 1979: Optimal Filtering. Prentice-Hall, 367 pp.

Anderson, J. L., 2001: An ensemble adjustment Kalman filter for data assimilation. Mon. Wea. Rev., 129, 2884-2903, https://doi.org/ 10.1175/1520-0493(2001)129<2884:AEAKFF $>2.0 . C O ; 2$.

Benny, A. H., and G. J. Dawson, 1983: Satellite imagery as an aid to bathymetric charting in the Red Sea. Cartogr. J., 20, 5-16, https://doi.org/10.1179/caj.1983.20.1.5.

Bierwirth, P. N., T. J. Lee, and R. V. Burne, 1993: Shallow sea-floor reflectance and water depth derived by unmixing multispectral imagery. Photogramm. Eng. Remote Sens. 59, 331-338.

Birkemeier, W. A., and E. B. Thornton, 1994: The Duck94 nearshore field experiment. Coastal Dyn., 94, 815-821.

- H. C. Miller, S. D. Wilhelm, A. E. DeWall, and C. S. Gorbics, 1985: A user's guide to the Coastal Engineering Research Center's (CERC's) field research facility. U.S. Army Corps of Engineers Instruction Rep. CERC-85-1, 145 pp.

Brodie, K. L., M. L. Palmsten, T. J. Hesser, P. J. Dickhudt, B. Raubenheimer, H. Ladner, and S. Elgar, 2018: Evaluation of video-based linear depth inversion performance and applications using altimeters and hydrographic surveys in a wide range of environmental conditions. Coastal Eng., 136, 147 160, https://doi.org/10.1016/j.coastaleng.2018.01.003.

De Vries, S., D. Hill, M. De Schipper, and M. Stive, 2011: Remote sensing of surf zone waves using stereo imaging. Coastal Eng., 58, 239-250, https://doi.org/10.1016/ j.coastaleng.2010.10.004.

Evensen, G., 2003: The ensemble Kalman filter: Theoretical formulation and practical implementation. Ocean Dyn., 53, 343 367, https://doi.org/10.1007/s10236-003-0036-9. 2009: Data Assimilation: The Ensemble Kalman Filter. Springer Science and Business, $332 \mathrm{pp}$.

Ghorbanidehno, H., A. Kokkinaki, J. Y. Li, E. Darve, and P. K. Kitanidis, 2015: Real-time data assimilation for large-scale systems: The spectral Kalman filter. Adv. Water Resour., 86, 260-272, https://doi.org/10.1016/j.advwatres.2015.07.017.

, _— , P. K. Kitanidis, and E. Darve, 2017: Optimal estimation and scheduling in aquifer management using the rapid feedback control method. Adv. Water Resour., 110, 310-318, https://doi.org/10.1016/j.advwatres.2017.10.011.

Halko, N., P.-G. Martinsson, and J. A. Tropp, 2011: Finding structure with randomness: Probabilistic algorithms for constructing approximate matrix decompositions. SIAM Rev., 53, 217-288, https://doi.org/10.1137/090771806.

Hamill, T. M., and C. Snyder, 2000: A hybrid ensemble Kalman filter-3D variational analysis scheme. Mon. Wea. Rev., 128, 2905-2919, https://doi.org/10.1175/1520-0493(2000)128<2905: $\mathrm{AHEKFV}>2.0 . \mathrm{CO} ; 2$.

Hathaway, K., and C. Long, 2008: A comparison of directional wave measurements from an $\mathrm{ADCP}, \mathrm{AWAC}$, and pressure 
sensor array. Eos, Trans. Amer. Geophys. Union, (Fall Meeting Suppl.), 89, Abstract OS13D-1244.

Holland, K., J. Puleo, and T. Kooney, 2001: Quantification of swash flows using video-based particle image velocimetry. Coastal Eng., 44, 65-77, https://doi.org/10.1016/S03783839(01)00022-9.

Holman, R., and M. C. Haller, 2013: Remote sensing of the nearshore. Annu. Rev. Mar. Sci., 5, 95-113, https://doi.org/10.1146/ annurev-marine-121211-172408.

, T. C. Lippmann, P. V. O'Neill, and K. Hathaway, 1991: Video estimation of subaerial beach profiles. Mar. Geol., 97, 225-231, https://doi.org/10.1016/0025-3227(91)90028-3.

— N. N. Plant, and T. Holland, 2013: Cbathy: A robust algorithm for estimating nearshore bathymetry. J. Geophys. Res. Oceans, 118, 2595-2609, https://doi.org/10.1002/jgrc.20199.

Houtekamer, P. L., and H. L. Mitchell, 2005: Ensemble Kalman filtering. Quart. J. Roy. Meteor. Soc., 131, 3269-3289, https:// doi.org/10.1256/qj.05.135.

Hunt, B. R., E. J. Kostelich, and I. Szunyogh, 2007: Efficient data assimilation for spatiotemporal chaos: A local ensemble transform Kalman filter. Physica D, 230, 112-126, https://doi.org/ 10.1016/j.physd.2006.11.008.

Irish, J. L., and W. J. Lillycrop, 1999: Scanning laser mapping of the coastal zone: The SHOALS system. ISPRS J. Photogramm. Remote Sens., 54, 123-129, https://doi.org/10.1016/S0924-2716(99) 00003-9.

Kalman, R. E., 1960: A new approach to linear filtering and prediction problems. J. Basic Eng., 82, 35-45, https://doi.org/ 10.1115/1.3662552.

Kepert, J. D., 2004: On ensemble representation of the observationerror covariance in the ensemble Kalman filter. Ocean Dyn., 54, 561-569, https://doi.org/10.1007/s10236-004-0104-9.

Khodayi-mehr, R., W. Aquino, and M. M. Zavlanos, 2017: Modelbased active source identification in complex environments. arXiv, https://arxiv.org/abs/1706.01603.

$\longrightarrow, \ldots$, and 2018a: Distributed reduced order source identification. American Control Conf., Milwaukee, WI, IEEE, 1084-1089.

—_, Y. Kantaros, and M. M. Zavlanos, 2018b: Distributed state estimation using intermittently connected robot networks. arXiv, https://arxiv.org/abs/1805.01574.

Kitanidis, P. K., 2015: Compressed state Kalman filter for large systems. Adv. Water Resour., 76, 120-126, https://doi.org/10.1016/ j.advwatres.2014.12.010.

_ proach for large dimensional inverse problems. Water Resour. Res., 50, 5428-5443, https://doi.org/10.1002/2013WR014630.

Lee, J., and P. K. Kitanidis, 2014: Large-scale hydraulic tomography and joint inversion of head and tracer data using the principal component geostatistical approach (PCGA). Water Resour. Res., 50, 5410-5427, https://doi.org/10.1002/ 2014WR015483.

— H. Hhorbanidehno, M. W. Farthing, T. J. Hesser, E. F. Darve, and P. K. Kitanidis, 2018: Riverine bathymetry imaging with indirect observations. Water Resour. Res., 54, 3704-3727, https://doi.org/10.1029/2017WR021649.

Li, J. Y., A. Kokkinaki, H. Ghorbanidehno, E. F. Darve, and P. K. Kitanidis, 2015: The compressed state Kalman filter for nonlinear state estimation: Application to large-scale reservoir monitoring. Water Resour. Res., 51, 9942-9963, https://doi.org/ 10.1002/2015WR017203.

Li, Y., A. Kokkinaki, E. F. Darve, and P. K. Kitanidis, 2017: Smoothing-based compressed state Kalman filter for joint state-parameter estimation: Applications in reservoir characterization and $\mathrm{CO}_{2}$ storage monitoring. Water Resour. Res., 53, 7190-7207, https://doi.org/10.1002/2016WR020168.

Liu, H., D. Sherman, and S. Gu, 2007: Automated extraction of shorelines from airborne light detection and ranging data and accuracy assessment based on Monte Carlo simulation. J. Coastal Res., 23, 1359-1369, https://doi.org/10.2112/ 05-0580.1.

Lyzenga, D. R., 1985: Shallow-water bathymetry using combined lidar and passive multispectral scanner data. Int. J. Remote Sens., 6, 115-125, https://doi.org/10.1080/ 01431168508948428.

_ N N. P. Malinas, and F. J. Tanis, 2006: Multispectral bathymetry using a simple physically based algorithm. IEEE Trans. Geosci. Remote Sens., 44, 2251-2259, https://doi.org/10.1109/ TGRS.2006.872909.

Massey, T. C., M. E. Anderson, J. M. Smith, J. Gomez, and R. Jones, 2011: STWAVE: Steady-state spectral wave model user's manual for STWAVE, version 6.0. U.S. Army Corps of Engineers Rep. ERDC/CHL TR-11-1, 90 pp.

Mobley, C. D., L. K. Sundman, C. O. Davis, J. H. Bowles, T. V. Downes, R. A. Leathers, and E. M. Louchard, 2005: Interpretation of hyperspectral remote-sensing imagery by spectrum matching and look-up tables. Appl. Opt., 44, 35763592, https://doi.org/10.1364/AO.44.003576.

Möhrlen, C., and J. U. Jørgensen, 2009: A new algorithm for upscaling and short-term forecasting of wind power using ensemble forecasts. Proc. Eighth Int. Workshop on Large Scale Integration of Wind Power and on Transmission Networks for Offshore Wind Farms, Bremen, Germany, Energynautics, 15.

Morris, J., 2013: Estimation of the nearshore bathymetry using remote sensing techniques. M.S. thesis, Dept. of Civil Engineering and Geosciences, Delft University of Technology, $132 \mathrm{pp}$.

Nenna, V., A. Pidlisecky, and R. Knight, 2011: Application of an extended Kalman filter approach to inversion of time-lapse electrical resistivity imaging data for monitoring recharge. Water Resour. Res., 47, W10525, https://doi.org/10.1029/ 2010WR010120.

Ott, E., and Coauthors, 2004: A local ensemble Kalman filter for atmospheric data assimilation. Tellus, 56A, 415-428, https:// doi.org/10.1111/j.1600-0870.2004.00076.x.

Pham, D. T., J. Verron, and M. C. Roubaud, 1998: A singular evolutive extended Kalman filter for data assimilation in oceanography. J. Mar. Syst., 16, 323-340, https://doi.org/10.1016/ S0924-7963(97)00109-7.

Puleo, J. A., G. Farquharson, S. J. Frasier, and K. T. Holland, 2003: Comparison of optical and radar measurements of surf and swash zone velocity fields. J. Geophys. Res., 108, 3100, https:// doi.org/10.1029/2002JC001483.

Rao, K. R., and P. Yip, 2014: Discrete Cosine Transform: Algorithms, Advantages, Applications. Academic Press, 512 pp.

Sallenger, A. H., Jr., W. B. Krabill, R. N. Swift, J. Brock, J. List, M. Hansen, and K. Morgan, 2003: Evaluation of airborne topographic lidar for quantifying beach changes. J. Coastal Res., 19, 125-133.

Sandidge, J. C., and R. J. Holyer, 1998: Coastal bathymetry from hyperspectral observations of water radiance. Remote Sens. Environ., 65, 341-352, https://doi.org/10.1016/S0034-4257(98) 00043-1.

Sasso, R., 2012: Video-based nearshore bathymetry estimation for rip current forecasting on a macrotidal beach. M.S. thesis, 
Dept. of Civil Engineering and Geosciences, Delft University of Technology, $181 \mathrm{pp}$.

Sherman, J., and W. J. Morrison, 1950: Adjustment of an inverse matrix corresponding to a change in one element of a given matrix. Ann. Math. Stat., 21, 124-127, https://doi.org/10.1214/ aoms/1177729893.

Smith, J. M., 2001: Modeling nearshore wave transformation with STWAVE. U.S. Army Corps of Engineers Tech. Rep. ERDC/ CHL CHETN-I-64, 10 pp.

—, A. R. Sherlock, and D. T. Resio, 2001: STWAVE: Steadystate spectral wave model user's manual for STWAVE, version 3.0. U.S. Army Corps of Engineers Tech. Rep. ERDC/ CHL SR-01-1, 81 pp.

Stockdon, H. F., and R. A. Holman, 2000: Estimation of wave phase speed and nearshore bathymetry from video imagery. J. Geophys. Res., 105, 22 015-22 033, https://doi.org/10.1029/ 1999JC000124.

Strang, G., 1999: The discrete cosine transform. SIAM Rev., 41, 135-147, https://doi.org/10.1137/S0036144598336745.

Tahmasebi, P., 2017: HYPPS: A hybrid geostatistical modeling algorithm for subsurface modeling. Water Resour. Res., 53, 5980-5997, https://doi.org/10.1002/2017WR021078.

_- and M. Sahimi, 2013: Cross-correlation function for accurate reconstruction of heterogeneous media. Phys. Rev. Lett., 110, 078002, https://doi.org/10.1103/PhysRevLett.110.078002.

- and S. Kamrava, 2018: A multiscale approach for geologically and flow consistent modeling. Transp. Porous Media, 124, 237-261, https://doi.org/10.1007/s11242-018-1062-x.
- and M. Sahimi, 2018: A stochastic multiscale algorithm for modeling complex granular materials. Granular Matter, 20, 45, https://doi.org/10.1007/s10035-018-0816-z.

—, , and M. G. Shirangi, 2018: Rapid learning-based and geologically consistent history matching. Transp. Porous Media, 122, 279-304, https://doi.org/10.1007/s11242-018-1005-6.

Tronvig, K. A., 2005: Near-shore bathymetry. Hydro Int., 9, 24-25.

Tsyrulnikov, M., 2009: Is the local ensemble transform Kalman filter suitable for operational data assimilation? COSMO Newsletter, No. 10, DWD, Offenbach, Germany, 22-36.

Van Dongeren, A., N. Plant, A. Cohen, D. Roelvink, M. C. Haller, and P. Catalan, 2008: Beach wizard: Nearshore bathymetry estimation through assimilation of model computations and remote observations. Coastal Eng., 55, 1016-1027, https://doi.org/ 10.1016/j.coastaleng.2008.04.011.

Wilson, G., and H. T. Özkan-Haller, 2012: Ensemble-based data assimilation for estimation of river depths. J. Atmos. Oceanic Technol., 29, 1558-1568, https://doi.org/10.1175/JTECH-D12-00014.1.

, - - and R. A. Holman, 2010: Data assimilation and bathymetric inversion in a two-dimensional horizontal surf zone model. J. Geophys. Res., 115, C12057, https://doi.org/ 10.1029/2010JC006286.

$-\longrightarrow,-$ M. C. Haller, D. A. Honegger, and C. C. Chickadel, 2014: Surf zone bathymetry and circulation predictions via data assimilation of remote sensing observations. J. Geophys. Res. Oceans, 119, 1993-2016, https://doi.org/ 10.1002/2013JC009213. 